\title{
Numerical Modeling of Thermal Behaviors and Vertical Motions of Polar Patrol Balloon with Auto-Ballasting System
}

\author{
Akira KADOKURA \\ National Institute of Polar Research, Tokyo 173, Japan
}

(Received February 23, 1994; Revised December 7, 1994; Accepted January 20, 1995)

\begin{abstract}
A time-dependent numerical model has been developed to investigate vertical motions of a zero-pressure balloon with an auto-ballasting system. This model is applied to the data of a Polar Patrol Balloon (PPB) experiment in January 1990, and the following results are obtained: (a) The balloon behaviors can be described by four periods in a day (post-noon descending, night ballasting, morning ascending, and pre-noon expulsion) and three stages during a flight (ballasting without sunset, ballasting with sunset, and no ballasting with sunset). (b) Two main factors determine the daily ballasting amount. One is the noonmidnight difference of the temperature ratio between the interior gas and the ambient air, and the other is the weight of the interior gas. The ballasting amount increases as the balloon approaches the latitude region where sunset exists, and decreases after it enters the region. (c) The vertical motions and the daily ballasting amount are strongly influenced by the radiative properties of the interior gas, especially by its absorptivity for the visible wave radiation. (d) While the auto-ballast control is operated, the maximum altitude of the balloon increases day by day, and after the control is terminated, both the maximum and minimum altitudes decrease day by day. These altitude changes can be explained by the ballasting and leakage of the interior gas.
\end{abstract}

Differences between the model calculations and the observations are also discussed.

\section{Introduction}

"Polar Patrol Balloon (PPB)" is a name of a stratospheric zero-pressure balloon equipped with a flight level control system (auto-ballasting system), which is launched from an Antarctic station in austral summer and characterized by its long duration, circumpolar trajectory and recurrence. The purpose of this paper is to construct a numerical model to investigate the thermal and dynamic behaviors of PPB responding to the changes of external radiative conditions, ballasting, gas expulsion, etc. Since the time resolution of the observational data, obtained by ARGOS satellite link system, is too coarse to analyze the balloon motion in detail, it is necessary to take a numerical approach for this investigation. Another merit of such a numerical approach is that it permits a prediction for the results of a future PPB plan under various launching and trajectory conditions.

Quantitative investigations on the thermal and dynamic behaviors of a stratospheric balloon have been made for many years. Nishimura et al. (1973) analyzed various thermal processes to determine the interior gas temperature of a balloon and presented a method to calculate the vertical velocity of the balloon at a given height. They also showed the contribution of each thermal process to the change of the gas temperature. They treated two time-independent cases, (perpetual day and night). Nishimura (1990) analyzed the motion of a stratospheric balloon launched in Antarctica with the same method. Lally (1983) did a similar approach for his proposed RACOON (radiation controlled balloon) system with assuming no free lift nor ballasting.

Kreith and Kreider (1974) developed a time-dependent numerical model for investigation of the performance of stratospheric balloons. In their model, they considered the effects of 
various thermal processes as Nishimura et al. (1973) did, including the processes of gas expulsion, valving, and ballasting. Carlson and Horn (1981) developed another time-dependent model with assuming the interior gas to be heated by its finite absorption properties for the visible and IR wave radiations. They compared their model calculations with several experimental results concerning the flight level change and the temperature difference between the interior gas and the film, and demonstrated the validity and accuracy of their model.

Basically the same model as Carlson and Horn (1981) is applied to the PPB analysis in this paper. In addition to their formulation, auto-ballasting procedure and diffusion processes both for the interior gas and for the ambient air are considered. This numerical model calculates the vertical motion of $\mathrm{PPB}$, and its horizontal motion is given by the actual trajectory in a real experiment. The PPB experiment executed in January 1990 is chosen as the real experiment to be analyzed in this paper, because it accomplished a long duration flight without any launching and technological troubles and the balloon experienced various circumstances (ballasting without sunset, ballasting with sunset, and no ballasting with sunset) during the flight.

\section{PPB Experiment in January 1990}

The PPB experiment in January 1990 was executed by the 30th Japanese Antarctic Research Expedition (JARE-30) at Syowa Station in Antarctica. The purpose and the results of the experiment were introduced in detail in Kadokura et al. (1991). Figure 1 shows the trajectory of the PPB, and Fig. 2 shows the time variations of the observational data which were obtained by ARGOS satellite system. The first operation of the auto-ballasting system worked on the third day (January 8 ) of the flight. The ballasting amount increased from the eighth day (January 13) as the daily maximum zenith angle approached the geometrical sunset angle. It reached

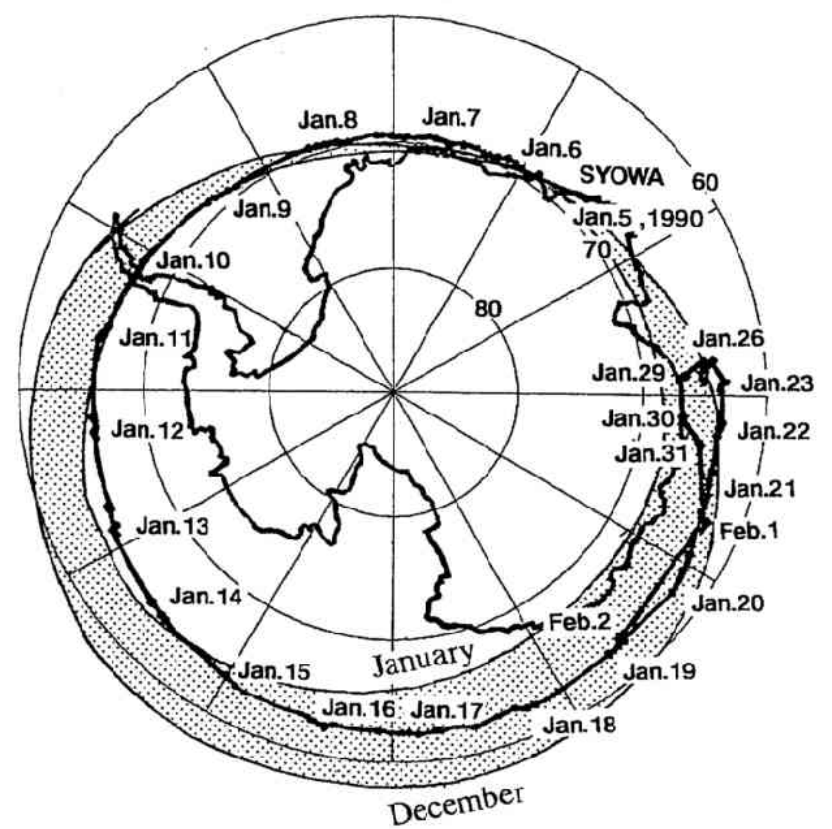

Fig. 1. Trajectory of the JARE-30 PPB (thick line). Shaded area corresponds to the region between the calculated monthly averaged wind stream lines at $31 \mathrm{~km}$ in January and in December from Yamanaka et al. (1988). This figure is a reproduction of Fig. 7 of Kadokura et al. (1991). 


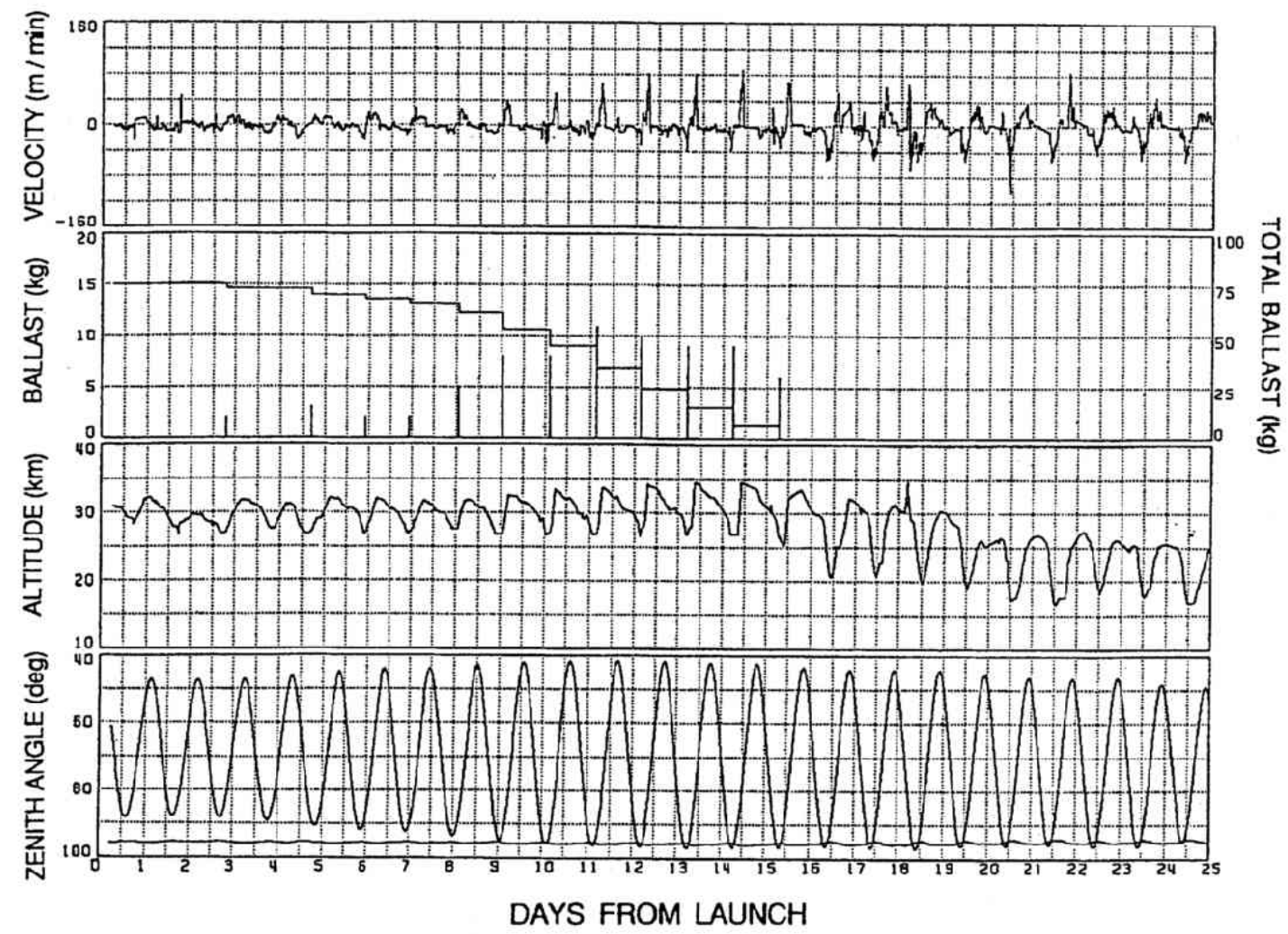

Fig. 2. Time variations of the observational data of JARE-30 PPB; Bottom: Calculated solar zenith angle at the balloon position. Solid line around $95^{\circ}$ shows the zenith angle of the geometrical sunset at the balloon position; Second from bottom: Altitude of the balloon, calculated from the data of a balloon-borne pressure gauge by using the monthly mean vertical profile of pressure at Syowa Station in January 1990, of which the standard levels are indicated in Fig. 3; Third: Spike- and step-like features show the ballasting amount and remaining total ballast amount, whose measures are shown in the left- and right-hand side, respectively; Top: Vertical velocity of the balloon.

a maximum on the 11th day (January 16), when there appeared a sunset time at the balloon position. On the 15th day (January 20), all the amount of the ballast installed in the payload was dropped, and then the auto-ballasting system could not work. Until the 15th day, the balloon altitude was maintained above the pre-set height of $27 \mathrm{~km}(20 \mathrm{mb})$ and the maximum altitude increased day by day (in particular, after the eighth day). After that, both the maximum and minimum altitudes decreased. The characteristics of the altitude change during the auto-ballast controlled period was apparently different from that of the un-controlled period. In the former period, the balloon moved upward with a high velocity after each ballasting, stopped suddenly at maximum height, and then gradually moved downward. In the latter period, the upward motion became gradual and the downward velocity became comparable to the upward one. After January 20 the balloon altitude decreased and the balloon began to experience westerly wind flows, hence its circumpolar drift motion was gradually slowed down. After January 29 it stayed in the westerly wind regime for nearly a whole day and eventually changed its drift direction. 


\section{Numerical Model of PPB}

\subsection{Basic equations}

Basic equations considered in this model are as follows:

3.1.1 Equation of motion of the balloon in the vertical direction

$$
\begin{aligned}
\left(m_{g a s}+m_{a i r, i n}+M+\frac{m_{a i r, o u t}}{2}\right) \frac{d v_{\perp}}{d t}= & \left(m_{a i r, o u t}-m_{a i r, i n}-m_{g a s}\right) g-M g \\
& -\frac{1}{2} C_{0} \rho_{a i r} S v_{\perp} \mathbf{v}_{\perp}
\end{aligned}
$$

where $m_{\text {gas }}$ and $m_{\text {air,in }}$ are the weights of the gas and the air inside the balloon, $m_{a i r, o u t}$ is the weight of the ambient air displaced by the balloon volume, $M$ is the total weight including the payload and the balloon, $v_{\perp}$ is the vertical velocity of the balloon, $C_{0}$ is the drag coefficient, $\rho_{\text {air }}$ is the density of the ambient air, $S$ is the cross section of the balloon, and $g$ is the gravitational constant. In the right-hand side of Eq. (1), each term from left denotes the lifting force, gravity, and air drag, respectively. The fourth term on the left-hand side denotes a virtual mass.

3.1.2 Thermal equation of the balloon film

$$
\begin{aligned}
& C p_{s} \rho_{s} d_{s} S_{B} \frac{d T_{f}}{d t}=-4 \pi r_{B}^{2} \kappa_{e f f} \sigma T_{f}^{4} \\
& \text { (IR emission loss } \\
& \text { : } \text { HRloss }, \text { film }_{\text {I }} \\
& +\pi r_{B}^{2} \varepsilon_{e f f} A_{p} J_{0} \\
& \text { (Direct solar radiation input } \quad: H_{\text {sol,film }} \text { ) } \\
& +\pi r_{B}^{2} \varepsilon_{e f f} A_{A} J_{0} \\
& \text { (Albedo input } \\
& \text { : } \left.H_{\text {albedo,film }}\right) \\
& +2 \pi r_{B}^{2} \kappa_{e f f}(1+e) J_{1} \\
& \text { (IR radiation from the earth }: H_{\text {IRinput,film }} \text { ) } \\
& +4 \pi r_{B}{ }^{2} \kappa_{i n t} \sigma\left(T_{g}{ }^{4}-T_{f}{ }^{4}\right) \\
& \text { (IR interchange with the gas }: H_{\text {IRint }} \text { ) } \\
& +4 \pi r_{B}^{2} a_{1} \frac{K_{g a s}}{2 r_{B}} N u_{n, i n}\left(T_{g}-T_{f}\right) \\
& \text { (Interior natural convection } \quad: H_{n, c o n v, i n} \text { ) } \\
& +4 \pi r_{B}^{2} a_{2} \frac{K_{a i r}}{2 r_{B}} N u_{n, \text { out }}\left(T_{\text {air }, \text { out }}-T_{f}\right) \\
& \text { (Ambient natural convection } \left.: H_{n, c o n v, o u t}\right) \\
& +4 \pi r_{B}^{2} a_{3} \frac{K_{\text {air }}}{2 r_{B}} N u_{f, \text { out }}\left(T_{\text {air }, \text { out }}-T_{f}\right) \\
& \text { (Ambient forced convection }: H_{f, c o n v, o u t} \text { ) }
\end{aligned}
$$

where $C p_{s}, \rho_{s}, d_{s}$, and $S_{B}$ are the specific heat at constant pressure, density, thickness, and surface area of the balloon film, respectively. $T_{f}, T_{g}$, and $T_{a i r, o u t}$ are the temperatures of the film, interior gas, and ambient air, respectively. $r_{B}$ is the radius of the balloon, assuming a spherical shape. $\kappa_{\text {eff }}$ and $\varepsilon_{\text {eff }}$ are the effective IR emissivity and effective solar absorptivity of the film, considering multiple radiation transmission, reflection, and absorption occurring between the film and the interior gas. $\kappa_{i n t}$ is the effective IR emissivity for interchange process with the gas. $A_{p}$ and $A_{A}$ are the transmittances of the direct and albedo solar radiation through the air, respectively. $J_{0}$ is the solar radiation at the top of the atmosphere. $J_{1}$ is the IR radiation flux from the earth, and $e$ is a coefficient representing the height dependence of the flux. $\sigma$ is the Stefan-Boltzmann constant. Each of $a_{1}, a_{2}$, and $a_{3}$ is the coefficient of effective surface for each convection. $K_{\text {gas }}$ and $K_{a i r}$ are the thermal conductivities of the interior gas and the ambient air, respectively. Each of $N u_{n, i n}, N u_{n, o u t}$, and $N u_{f, o u t}$ is the Nusselt number for each convection. Expressions or values of these variables or constants are described in detail in Appendix. 


\subsubsection{Thermal equation of the interior gas}

$$
\begin{aligned}
& C p_{g} m_{g a s} \frac{d T_{g}}{d t}=-4 \pi r_{B}{ }^{2} \kappa_{g, e f f} \sigma T_{g}{ }^{4} \\
& \text { (IR emission loss } \\
& \text { : } \left.H_{\text {IRloss,gas }}\right) \\
& +\pi r_{B}^{2} \varepsilon_{g, e f f} A_{p} J_{0} \\
& \text { (Direct solar radiation input } \quad: H_{\text {sol,gas }} \text { ) } \\
& +\pi r_{B}^{2} \varepsilon_{g, e f f} A_{A} J_{0} \\
& \text { (Albedo radiation input } \\
& \text { : } \left.H_{\text {albedo,gas }}\right) \\
& +2 \pi r_{B}^{2} \kappa_{g, e f f}(1+e) J_{1} \\
& \text { (IR radiation from the earth } \\
& \text { : H } \text { IRinput,gas }) \\
& -4 \pi r_{B}^{2} \kappa_{i n t} \sigma\left(T_{g}^{4}-T_{f}^{4}\right) \\
& \text { (IR interchange with the film }: H_{\text {IRint }} \text { ) } \\
& -4 \pi r_{B}^{2} a_{1} \frac{K_{g a s}}{2 r_{B}} N u_{n, i n}\left(T_{g}-T_{f}\right) \\
& +\frac{\gamma-1}{\gamma} C p_{g} m_{g a s} \frac{T_{g}}{P_{B}} \frac{d P_{g}}{d t} \\
& \text { (Interior natural convection } \\
& \text { : } \left.H_{n, c o n v, i n}\right) \\
& \text { (Adiabatic change } \\
& \text { : } \left.H_{\text {adia }}\right)
\end{aligned}
$$

where $\kappa_{g, \text { eff }}$ and $\varepsilon_{g, \text { eff }}$ are the effective IR emissivity and effective solar absorptivity of the gas, respectively. $\gamma$ and $C p_{g}$ are the specific heat ratio and the specific heat at constant pressure of the gas, respectively. $P_{g}$ is the interior gas pressure.

\subsubsection{Weight change equation of the interior gas}

$$
\frac{d m_{g a s}}{d t}=-\sqrt{\frac{P_{g}-P_{B}}{\left(l_{D} S_{D}+V_{f u l l}\right)\left(m_{g a s}+m_{a i r, i n}\right)}} S_{D} m_{g a s}-D_{g} \frac{n_{g}}{l_{D}} S_{D} m_{g a s, m o l}
$$

where $P_{B}$ is the ambient pressure, $l_{D}$ and $S_{D}$ are the length and cross section of the venting duct, respectively, $V_{f u l l}$ is the maximum volume of the balloon, $D_{g}$ is the diffusion coefficient of the gas, and $n_{g}$ and $m_{g a s, m o l}$ are the number density and the weight of the gas molecule, respectively. The first term in the right-hand side of Eq. (4) denotes the expulsion process and the second term, the diffusion process. Only when the interior gas pressure becomes greater than the ambient pressure, does expulsion process occur.

3.1.5 Weight change equation of the interior air

$$
\begin{aligned}
\frac{d m_{a i r, i n}}{d t}= & -\sqrt{\frac{P_{g}-P_{B}}{\left(l_{D} S_{D}+V_{f u l l}\right)\left(m_{\text {gas }}+m_{a i r, i n}\right)}} S_{D} m_{a i r, i n} \\
& +D_{a i r} \frac{\left(n_{a i r, o u t}-n_{a i r, i n}\right)}{l_{D}} S_{D} m_{a i r, m o l}
\end{aligned}
$$

where $D_{\text {air }}$ is the diffusion coefficient of the air, $n_{\text {air, out }}$ and $n_{\text {air, in }}$ are the number density of the ambient air and the interior air, respectively, and $m_{a i r, m o l}$ is the weight of the air molecule. The first term in the right-hand side of Eq. (5) expresses the expulsion of the interior air and the second term, the diffusion of the ambient air.

\subsection{Radiative properties of the film and gas}

As shown by earlier investigations (e.g. Lally, 1983; Nishimura et al., 1973; Nishimura, 1990), one of the most important factors affecting the thermal behavior of the balloon is the radiative properties of the balloon film. Discrepancy between laboratory and real flight data has often been 
pointed out (e.g. Lally, 1983), and empirical values are frequently used. In the present model, the laboratory data measured by NASA/Langley in 1980 (Kiser, 1980) is used following Carlson and Horn (1981). It should be noted that according to the laboratory data, the solar absorptivity is about one fiftieth and the IR emissivity is about one sixth or one eleventh of the empirical values.

IR emissivity of the interior gas is expressed following Carlson and Horn (1981), which magnitude is about two orders smaller than the IR emissivity of the film. Hence $H_{\text {IRint }}$ in Eq. (2) and $H_{\text {IRloss,gas }}, H_{\text {IRinput,gas, }}$, and $H_{\text {IRint }}$ in Eq. (3) are negligibly small compared with other terms. Concerning the solar absorptivity of the gas, present model treats it as an unknown parameter, and various values are tested to find a most favorable value to give a result consistent with observation. Since helium, used as the lifting gas in a real experiment, is completely transparent to any radiation, this model assumes that some contaminant having such radiative properties is included inside the balloon, following Carlson and Horn (1981).

\subsection{Drag coefficient}

In the present model, the air drag coefficient is calculated from the Reynolds number by using the laboratory data for a sphere-shaped object introduced in Landau and Lifshitz (1954). Discrepancies between laboratory values and experimental results were often found, and empirical values were frequently used in the analysis of balloon motion (e.g. Nishimura et al., 1973; Carlson and Horn, 1981). Since the real shape of a balloon is non-spherical and it is known that there is a natural turbulent flow in the stratosphere which causes the eddy viscosity, the laboratory data may not be directly applicable. In Section 5, it will be discussed whether any discrepancies between the calculation and the observational results can be found.

\subsection{Atmospheric model}

The variation in atmospheric properties $\left(T_{\text {air }, \text { out }}\right.$ and $\left.P_{B}\right)$ with altitude is very important, since these properties directly affect balloon lift, drag, and heat transfer. For the atmospheric parameters, the present model uses the monthly mean values obtained from the upper-air observations at Syowa Station in January 1990, which are shown in Fig. 3. $T_{\text {air,out }}\left(P_{B}\right)$ is calculated by a linear (exponential) interpolation with altitude from the observational data. For the higher altitudes above $15 \mathrm{mb}$, these parameters are calculated by a linear and exponential extrapolation from the values at $15 \mathrm{mb}$ and $20 \mathrm{mb}$. These inter- and extra-polated values are shown by a straight line in Fig. 3. A different temperature-altitude relation shown by a break line in Fig. 3 is also tested, which is derived by a least square fit to the data above $200 \mathrm{mb}$. Differences between the calculated results using these two different temperature-altitude relations will be discussed in Section 5. This simple treatment is considered to be valid for the summer season (easterly wind period) in the present analysis, because it is known that there are no planetary-scale strong disturbances in the stratosphere and the atmospheric properties are almost uniform in the zonal direction (Yamanaka et al., 1988). However, for the winter season, this treatment may be invalid because there are strong planetary-scale disturbances in the stratosphere. For more precise modeling applicable to all seasons, atmospheric properties at each location and at each time should be calculated from world-wide meteorological data (e.g. from the objective analysis data of Japan Meteorological Agency).

\subsection{Albedo and IR radiation from the earth}

In the present model, the albedo of the earth surface is set in a constant value of $0.5(0.65)$ for the latitude greater (less) than $-70^{\circ}$, which is based on an analysis of satellite data (Hartmann et al., 1986). Since the balloon latitude was almost always greater than $-70^{\circ}$ throughout the flight (see Fig. 1), this simplification implies that the albedo below the balloon is nearly constant at 0.5 except for the first few days. In the real case, the albedo significantly depends on the distributions of the snow, open-sea, sea-ice, and clouds below the balloon. The local and temporal variation 


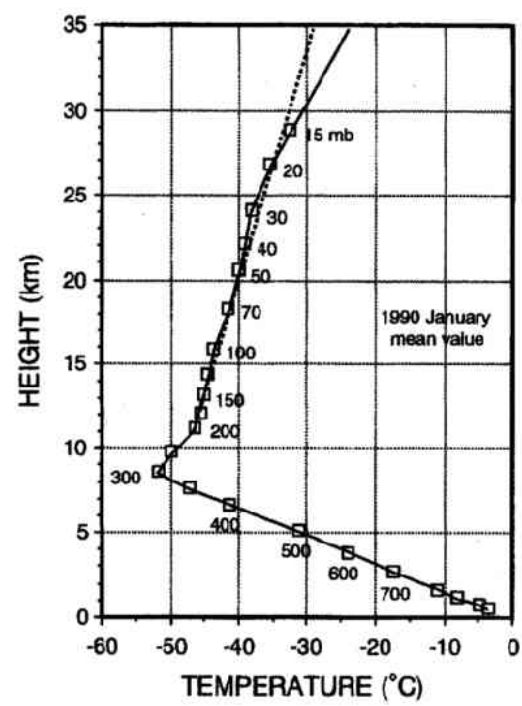

Fig. 3. Square symbols: Monthly averaged height profile of the air temperature observed at Syowa Station at 00 UT in January 1990; Straight line: Linearly inter- and extra-polated values from the data; Break line: Least square fit line to the data above $200 \mathrm{mb}$.

of the distribution is not considered in this model, and may cause a discrepancy between the calculation and the observational result, which will be discussed in Section 5 . The IR radiation from the earth is set in a constant value of $0.5 \times 10^{-2} \mathrm{cal} / \mathrm{cm}^{2} \mathrm{~s}$ (about $210 \mathrm{~J} / \mathrm{m}^{2} \mathrm{~s}$ ), referring to Hartmann et al. (1986). In the real case, there is a local and temporal variation in this value, depending on the surface temperature of the earth and the presence of clouds.

\subsection{Auto-ballasting procedure}

The altitude of a PPB is maintained above a preset height by the auto-ballast control. If the balloon altitude falls below the controlled height, a fixed amount of ballast is dropped at constant time intervals. For the JARE-30 PPB, the controlled height is $27 \mathrm{~km}(\sim 20 \mathrm{mb})$, the fixed amount of ballast is $1 \mathrm{~kg}$, and the constant time interval is 3 minutes. In the model, if the calculated altitude falls below $27 \mathrm{~km}$, the total weight is reduced by $1 \mathrm{~kg}$ at 3 minute interval, until the altitude increases above $27 \mathrm{~km}$. This auto-ballast control is not operated after the total amount of ballast has been dropped.

\subsection{Horizontal trajectory}

The model calculates the vertical motion of the balloon. In the horizontal plane, the model must trace the actual trajectory of the JARE-30 PPB shown in Fig. 1, because the latitudinal and longitudinal position at each time affects the thermal condition of the balloon. These horizontal positions are linearly interpolated in time from the observational data.

\subsection{Calculation procedure}

The Runge-Kutta method is used in the present calculation, with a time step $\Delta t$ of 0.25 minutes. The calculation starts at the time of launch at 08:24 UT, January 5, 1990. At launch it is assumed that $T_{f}=T_{a i r, o u t}, T_{g}=T_{a i r, o u t}$, and $m_{a i r, i n}=0$. The initial total weight is 189.7 $\mathrm{kg}$, with a ballast of $79 \mathrm{~kg}$; The free lift is $6 \mathrm{~kg}$. After 33 minutes from launch, $4 \mathrm{~kg}$ ballast is dropped, in accord with the actual experiment. From the position of the balloon at each time $t$, the solar zenith angle, atmospheric pressure and temperature can be calculated. Solving 
numerically Eqs. (1), (2), (3), (4), (5) together with $d h_{B} / d t=v_{\perp}$, one can obtain $v_{\perp}, T_{f}, T_{g}$, $m_{\text {gas }}, m_{a i r, i n}$, and the balloon altitude $h_{B}$ at the next time step $t+\Delta t$. If $h_{B}$ is less than 27 $\mathrm{km}$ and the previous ballasting occurred more than 3 min earlier, or previous $h_{B}$ is above $27 \mathrm{~km}$, then the total weight $M$ is reduced by $1 \mathrm{~kg}$. This auto-ballasting procedure is started 5 hours after launch and ends after the ballast payload $(79 \mathrm{~kg})$ has been dropped.

\section{Calculation Results}

\subsection{Overview and comparison with observations}

Figure 4 shows the calculation results corresponding to the observational results shown in Fig. 2. It can be seen from Fig. 4 that there are following three distinct stages in this experiment;

Stage 1 : ballasting without sunset (until the tenth day), Stage 2 : ballasting with sunset Stage 3 : no ballasting with sunset (from the tenth day until the 15th day), (from the 15th day).

\subsubsection{Ballast drop}

In Stages 1 and 2, the ballasting amount increases from the eighth day, and the total amount of the ballast has been dropped on the 15th day. These features and the timing of each ballasting from the eighth day until the 15 th day are almost consistent with the observations. Compared more precisely with the observation as shown in Fig. 5 , the ballasting begins two days earlier, and reaches a maximum one day earlier. After the maximum day the ballasting amount decreases day by day for both results, though the calculation value is about $2 \mathrm{~kg}$ smaller than the observational one. The maximum day in the calculation corresponds to the day when sunset time first appears at the balloon altitude.

\subsubsection{Altitude}

In Stages 1 and 2, the altitude is maintained above about $27 \mathrm{~km}$ by the auto-ballast control, and the maximum altitude increases after each ballasting. The increasing rate becomes larger from the eighth day. In Stage 3, both the maximum and the minimum altitudes decrease day by day. These features are consistent with the observations. Figure 6 shows calculated daily maximum and minimum altitudes compared with those actually observed. In Stages 1 and 2, the increasing rate of the maximum altitude in the calculation is almost same as in the observation, and the maximum altitude on the 15 th day is almost same in both results. In Stage 3 , the decreasing rates of the maximum and minimum altitudes are almost same in both results, but the maximum-minimum altitude difference in the calculation is about $6.6 \mathrm{~km}$ larger than in the observation. In Stages 1 and 2, the calculated balloon in Fig. 4 keeps the maximum altitude for a relatively long period, while the actual balloon altitude shown in Fig. 2 decreases soon after reaching the maximum altitude.

\subsubsection{Vertical velocity}

In Stages 1 and 2, the balloon moves upward with a high velocity after each ballasting, and stops suddenly, and gradually moves downward as the solar zenith angle becomes larger. In Stage 3 , the upward motion of the balloon becomes gradual, and the downward one is also gradual except around the sunset time on each day. Around sunset, the downward velocity increases sharply. These features are consistent with the observations. Both the upward and downward velocities in Fig. 4 are larger than those in Fig. 2. In Stages 1 and 2, the upward peak value in Fig. 4 is 140-300\%, and in Stage 3 both the upward and downward ones are about $130 \%$ of those shown in Fig. 2. When the balloon stays at the maximum floating height, there is an oscillation in the velocity, as shown in Fig. 4.

\subsubsection{Temperatures}

In this model, variation of $T_{\text {air,out }}$ is directly corresponding to that of the balloon altitude. Accompanying the increase of $T_{a i r, o u t}$ at daytime in Stages 1 and 2, both $T_{f}$ and $T_{g}$ also increase, 

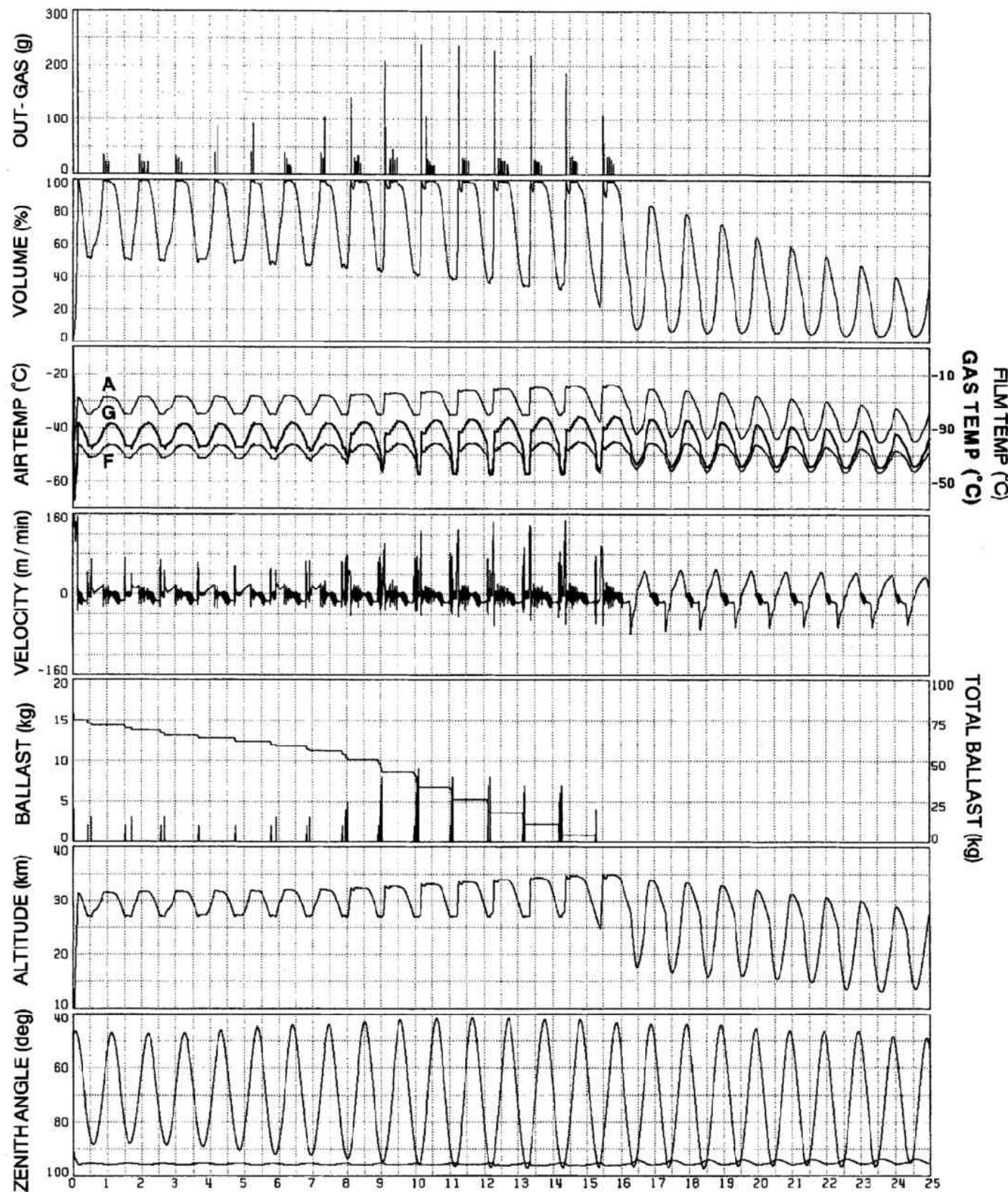

\section{DAYS FROM LAUNCH}

Fig. 4. Calculation results. Four panels from the bottom are corresponding to the panels in Fig. 2. The fifth panel from bottom shows the temperatures of $T_{a i r, o u t}, T_{f}$, and $T_{g}$, which are denoted as 'A', ' $F$ ', and ' $\mathrm{G}$ ', respectively. The measure for $T_{a i r, o u t}\left(T_{f}\right.$ and $\left.T_{g}\right)$ is indicated on the left-hand (right-hand) side. The sixth panel shows the volume of the balloon, expressed as a percentage relative to the maximum volume. Top panel shows the weight of the gas expelled from the balloon. 


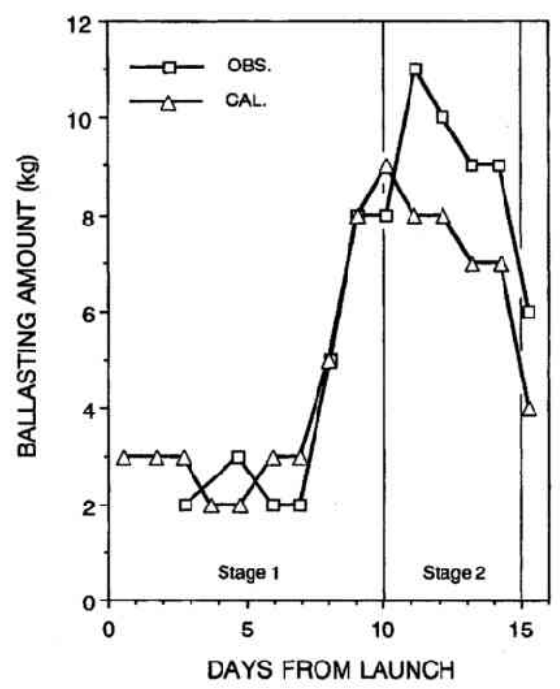

Fig. 5. Day-to-day variations of the ballasting amount in the calculation (triangles), compared with the observation (squares). Vertical line at the tenth day shows the day when sunset time first appears at the balloon altitude, and that at the 15 th day does the day when the total ballast payload has been dropped.

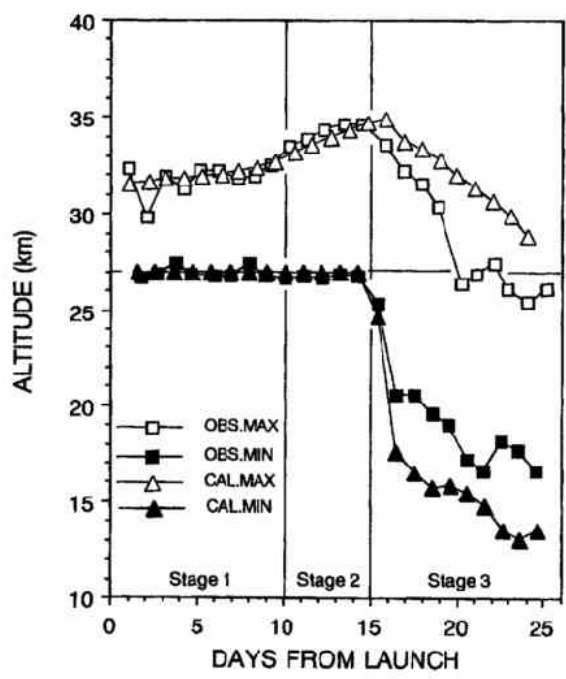

Fig. 6. Day-to-day variations of the maximum (white symbols) and the minimum (black symbols) altitude in the calculation (triangles), compared with the observation (squares). Horizontal line at $27 \mathrm{~km}$ shows the auto-ballast height.

and in Stage 3, these values decrease both at daytime and at nighttime. Figure 7(a) shows the time history of $T_{a i r, o u t}, T_{f}$, and $T_{g}$ at noon and at midnight. Throughout the computation period, the noon values of $T_{g}$ are nearly equal to $T_{a i r, o u t}$, and are always larger than $T_{f}$. In Stage 1, $T_{g}$ at midnight decreases sharply as the maximum solar zenith angle approaches to the sunset angle. On the tenth day, it becomes nearly equal to $T_{f}$ of about $-47^{\circ} \mathrm{C}$ and lower than $T_{a i r, o u t}$ by about 


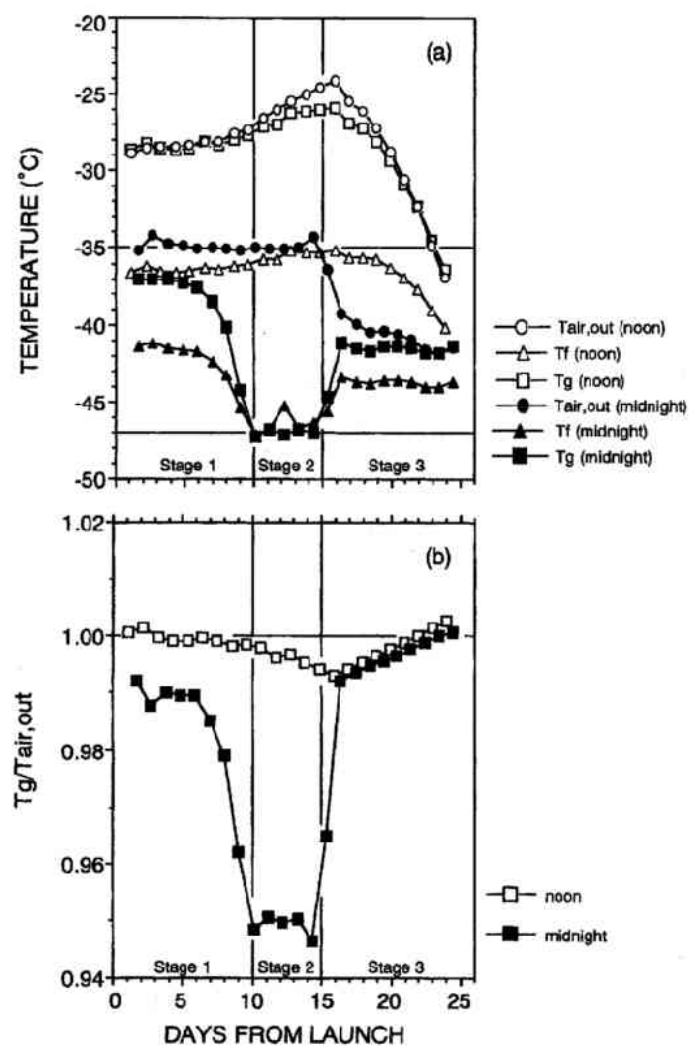

Fig. 7. Day-to-day variations of (a) $T_{\text {air,out }}$ (circles), $T_{f}$ (triangles), and $T_{g}$ (squares) in the calculation, at noon (white) and at midnight (black); and (b) temperature ratio $T_{g} / T_{\text {air, out }}$ at noon (white) and at midnight (black). In (a), horizontal line at $-35^{\circ} \mathrm{C}$ shows the atmospheric temperature at the auto-ballast height, and that at $-47^{\circ} \mathrm{C}$ indicates the midnight level of $T_{f}$ and $T_{g}$.

$12^{\circ} \mathrm{C}$. In Stage 2, relations among these temperatures are almost unchanged. In Stage 3 , the midnight values of $T_{g}$ become nearly equal to $T_{a i r, o u t}$, and larger than $T_{f}$ by about $2^{\circ} \mathrm{C}$. Since the measurements of $T_{\text {air,out }}, T_{f}$, and $T_{g}$ were not executed during the JARE-30 experiment, there are no observational results which can be compared with these calculated results.

4.1.5 Balloon volume

Balloon volume $V_{B}$ at each time is calculated from $m_{a i r, i n}, m_{g a s}, T_{g}$, and $P_{g}$ as follows,

$$
V_{B}=\left(\frac{m_{a i r, i n}}{M_{a i r}}+\frac{m_{g a s}}{M_{g a s}}\right) \frac{R T_{g}}{P_{g}}
$$

where $M_{a i r}$ and $M_{\text {gas }}$ are molecular weights of the air and the gas, respectively, and $R$ is the universal gas constant. The second panel from the top in Fig. 4 shows the time development of $V_{B}$ expressed as a percentage relative to the maximum volume $V_{f u l l}$. In Stages 1 and $2, V_{B}$ equals $V_{\text {full }}$ at highest altitude, and attains a much smaller minimum value for each lowest altitude, which gradually decreases day by day. In Stage 3, both the daily maximum and minimum value of $V_{B}$ decrease as the balloon altitudes decrease, and the maximum value never reaches $V_{f u l l}$.

\subsubsection{Interior gas and air weights}

In the top panel of Fig. 4, it can be seen that in Stages 1 and 2, gas expulsion occurs when $V_{B}$ reaches $V_{\text {full }}$. In Fig. 8, the time history of the interior gas weight is shown. The step-like 


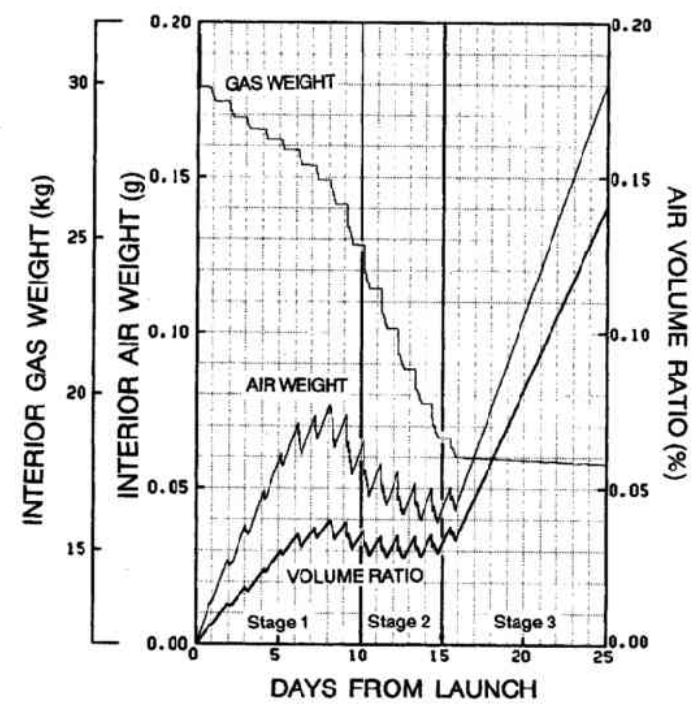

Fig. 8. Time history of the interior gas weight, interior air weight and volume ratio occupied by the interior air.

changes seen in Fig. 8 indicate that the expulsion process occurs. A large initial expulsion occurs when the balloon reaches a floating level after launch. After that, the expelled amount in each day is almost constant until the seventh day, increases from the eighth day, reaches a maximum on the 10th day, and gradually decreases until the 15th day. In Stage 3, the expulsion does not occur, and the interior gas weight decreases only by diffusion at a rate of about $23 \mathrm{~g} /$ day. This diffusion process occurs throughout the flight period.

Figure 8 also shows the time history of the weight and the occupied volume ratio of the interior air. The interior air weight increases because the ambient air diffuses into the balloon, and decreases because the interior air goes out by expulsion process. A sharp decrease, both in the weight and in the volume, shows that an expulsion process occurs. Until the eighth day, the increase by diffusion is greater than the decrease by expulsion, and from the eighth day until the 15 th day, the decrease is larger than the increase. In Stage 3, the expulsion process ceases, and the weight increases monotonously due to diffusion at a rate of about $15 \mathrm{~g} /$ day. Compared with the interior gas weight, the interior air weight is small by third to second order, and the volume occupied by the air is very small. Hence, its contributions to the total balloon mass and volume are negligible in the present analysis.

Though there are several differences between the calculated and observational results, it can be said that this model can simulate the behavior of the PPB enough well concerning the altitude variation and the ballasting process. Detailed process working on the PPB will be analyzed in the following sections based on this model calculation, and such differences as above mentioned will be discussed in Section 5 .

\subsection{Detailed process in each Stage}

Since the weight of the interior air $m_{\text {air,in }}$ is negligibly small compared with $m_{a i r, o u t}$ and $m_{\text {gas }}$, the lifting force $F$ is written as follows by using the Boyle-Charles law and the zero-pressure balloon condition;

$$
F=\left(m_{\text {air }, \text { out }}-m_{\text {gas }}\right) g
$$




$$
=m_{\text {gas }}\left(\frac{M_{\text {air }}}{M_{\text {gas }}} \frac{T_{g}}{T_{\text {air }, \text { out }}}-1\right) g .
$$

Hence, the free lift $f$, which is the difference between the lifting force and the gravity, is written as

$$
f=\left\{m_{\text {gas }}\left(\frac{M_{\text {air }}}{M_{\text {gas }}} \frac{T_{g}}{T_{a i r, o u t}}-1\right)-M\right\} g .
$$

The free lift determines the ascending-descending motion of the balloon. It can be seen from Eq. (8) that such vertical motion of the balloon is governed by the interior gas weight $m_{\text {gas }}$, temperature ratio $T_{g} / T_{\text {air,out }}$, and the total weight $M$.

\subsubsection{Stage 1: ballasting without sunset}

Figure 9 shows an example of the data in Stage 1 from the third day noon until the fourth day noon. Due to the solar radiation, $T_{g}$ becomes greater than $T_{f}$, and the natural convection between the gas and the film $H_{n, c o n v, \text { in }}$ is generated, which becomes the main input process (except $H_{\text {IRinput,film }}$ ) for the film and the main loss process for the interior gas.

At the third day noon, the balloon is in a floating condition with a nearly full volume, and the free lift $f$ is nearly equal zero. As the solar zenith angle increases, the interior gas is cooled ( $T_{g}$ decreases) mainly because the albedo radiation input $H_{\text {albedo,gas }}$ becomes small. The balloon volume is shrinking, lifting force decreases ( $f$ becomes negative), and then the balloon starts descending. As the balloon is descending, ambient air temperature $T_{\text {air,out }}$ decreases because of the altitude profile of the temperature in the stratosphere. Consequently, the ratio $T_{g} / T_{\text {air, out }}$ is almost unchanged, and $f$ keeps a small negative value during the descending period. The descending motion causes the adiabatic heating of the interior gas (increase of $H_{\text {adia }}$ ) and creates the ambient forced convection (increase of $H_{f, c o n v, o u t}$ ).

When the balloon descends below the controlled height $(27 \mathrm{~km})$, the auto-ballasting system works. At each ballasting, the total weight $M$ is reduced by $1 \mathrm{~kg}$. Since this amount is large enough to change $f$ to a positive value, the balloon is suddenly accelerated upward, and the interior gas is adiabatically cooled ( $T_{g}$ changes stepwise to a smaller value), which can be seen in the sharp reduction of $H_{\text {adia }}$. Because of the sharp reduction of $T_{g}, f$ becomes negative again after a short time, and the balloon moves downward again. This upward and downward motion occurs at each ballasting, and the altitude is maintained near the controlled height. Since $T_{\text {air, out }}$ is nearly constant, the ratio $T_{g} / T_{\text {air,out }}$ decreases at each ballasting, and becomes minimum at the last ballasting.

After the last ballasting before midnight, adiabatic cooling is not enough to cancel the upward acceleration. The upward velocity gradually decreases, but the balloon continues to ascend. After midnight, $H_{\text {albedo,gas }}$ increases, $T_{g}$ increases, the balloon volume is expanding, and the balloon is accelerated upward. As the balloon is ascending, $T_{a i r, \text { out }}$ also increases. Consequently, the ratio $T_{g} / T_{\text {air,out }}$ is maintained nearly constant during the ascending period, and $f$ keeps a small positive value. The ascending motion causes the adiabatic cooling of the interior gas (decrease of $H_{\text {adia }}$ ) and creates the ambient forced convection (increase of $H_{f, \text { conv,out }}$ ).

About 6 hours before the fourth day noon, the balloon volume becomes maximum, and the gas expulsion occurs. The interior gas is expelled so much that the free lift $f$ becomes slightly negative, and the balloon is suddenly decelerated, stops ascending, and starts moving downward. This deceleration causes adiabatic heating of the interior gas, and $T_{g}$ changes stepwise to a higher value. Because of the sharp increase of $T_{g}, f$ becomes slightly positive again after a short time, and the balloon moves upward again. This process is iterated several times, and the balloon oscillates vertically around the ceiling height with a few minute period, which is known as a hunting phenomenon. After the hunting motion, the balloon gradually moves downward. Because $H_{\text {albedo,gas }}$ is still increasing, $T_{g}$ gradually increases, and $f$ becomes positive again. Hence, the balloon moves upward again, and another hunting phenomenon occurs about 100 minutes after 


\section{Stage 1 : ballasting without sunset}

(a)

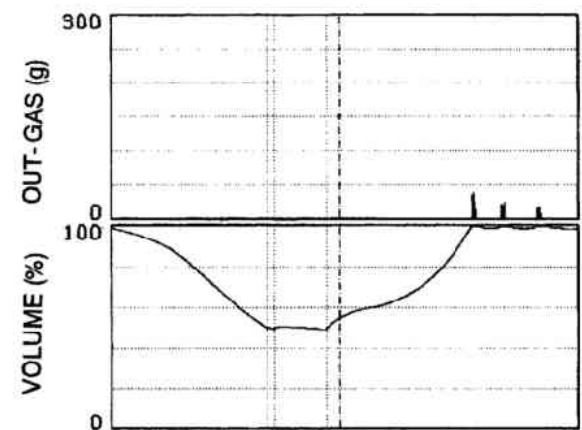

כั

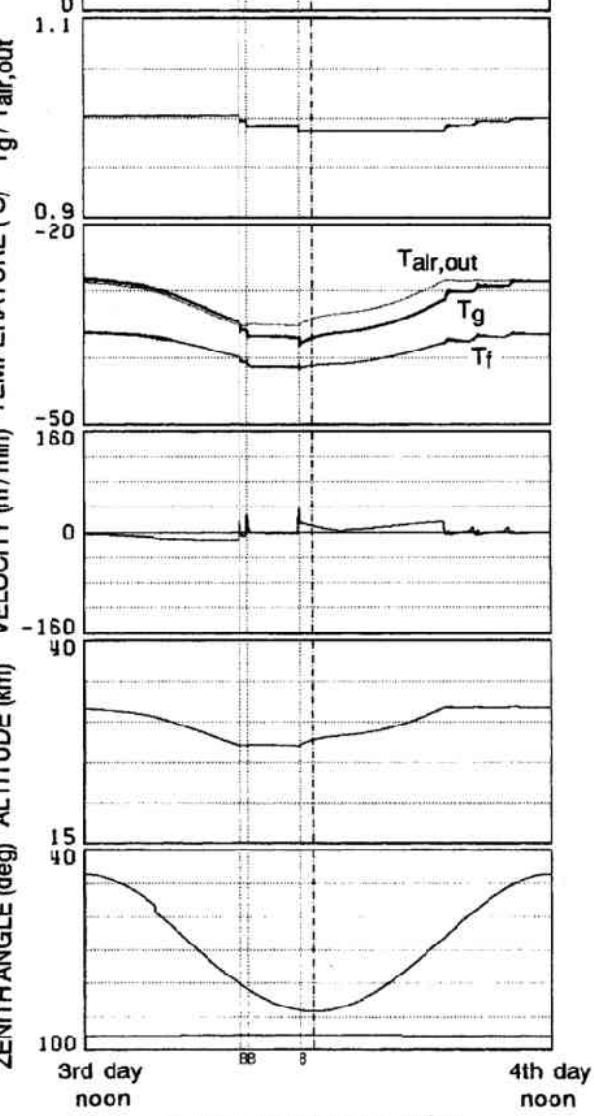

(b)

CONTRIBUTION OF EACH THERMAL PROCESS
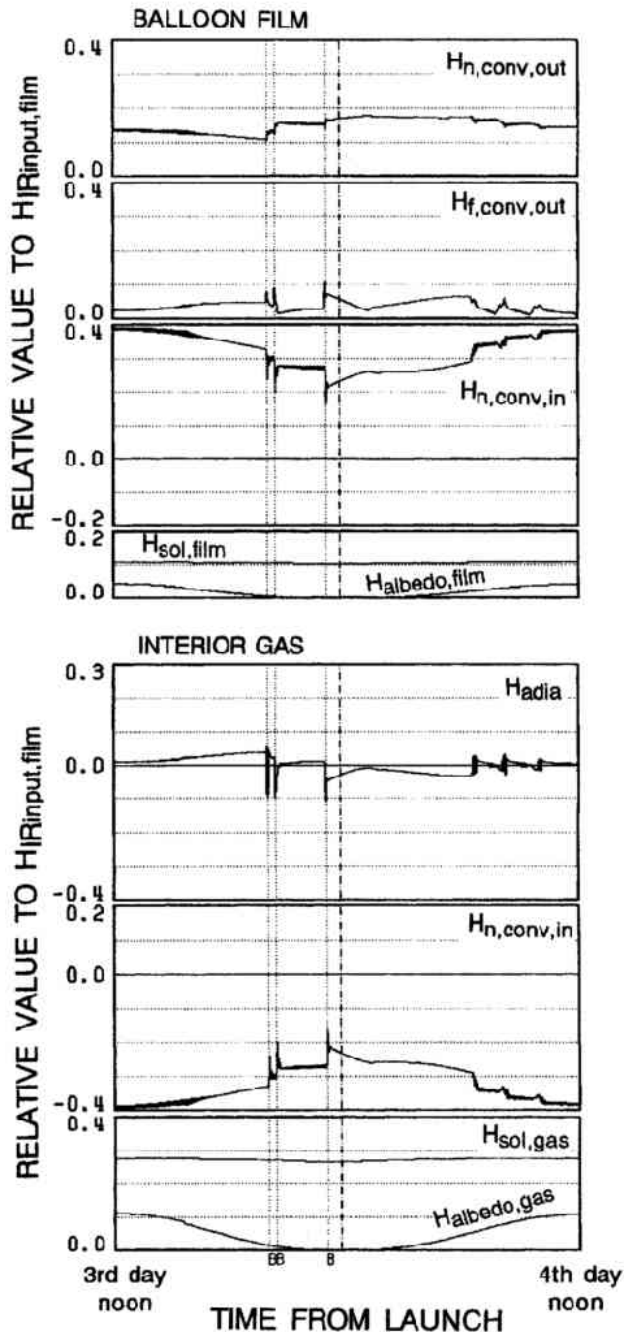

Fig. 9. An example of the data in Stage 1. Both abscissas of (a) and (b) represent time, from the third day noon until the fourth day noon. In (a), each panel from bottom shows the time development of the solar zenith angle, altitude, vertical velocity, temperatures of $T_{a i r, o u t}, T_{g}$, and $T_{f}$, temperature ratio of $T_{g} / T_{a i r, o u t}$, balloon volume, and the gas amount going out from the balloon, respectively. In (b), each panel shows the time development of the thermal energy flow of each thermal process for the balloon film (upper panels) and for the interior gas (lower panels). As mentioned in Subsection 3.2, $H_{\text {IRint }}, H_{I R l o s s, g a s}, H_{I \text { Rinput,gas }}$ are negligibly small and omitted from display. In (b), ordinate of each panel is the value relative to $H_{I R \text { Rinput, film }}$, and plus (minus) value means the input (loss) of the thermal energy flow. The dotted vertical line indicates the time of ballasting, and the dot-dash line denotes midnight. 
the previous one. There are several such hunting phenomena occurred until the next noon. Since $T_{\text {air, out }}$ is nearly constant, the ratio $T_{g} / T_{a i r, o u t}$ increases at each expulsion. After the last expulsion, the ratio becomes close to, but a little smaller than, the value at the previous noon.

\subsubsection{Stage 2: ballasting with sunset}

Figure 10 shows an example of the data in Stage 2, from the 10th day noon until the 11th day noon. Features before ballasting are similar to those in Stage 1. When the solar zenith angle becomes greater than $90^{\circ}$, the ground surface below the balloon enters the dark area, and the albedo radiation inputs $H_{\text {albedo,gas }}$ and $H_{\text {albedo,film }}$ become zero. Around that time, $H_{\text {sol,gas }}$ steeply decreases and becomes zero at the sunset time at the balloon altitude. Almost all ballastings occur before the sunset, and only one ballasting occurs during nighttime. Because of the decrease of $H_{s o l, g a s}$ and the adiabatic cooling at each ballasting, $T_{g}$ decreases, and $H_{n, c o n v, \text { in }}$ decreases until the sunset. During nighttime, both $H_{\text {sol,gas }}$ and $H_{\text {albedo,gas }}$ become zero, and the thermal processes effective to the gas are $H_{n, c o n v, \text { in }}$ and $H_{\text {adia }}$. Except at the time of ballasting, the adiabatic change $H_{a d i a}$ is small, and hence the interior convection $H_{n, c o n v, i n}$ is small, which means that $T_{g}$ is nearly equal to $T_{f}$ during nighttime. Because of the reduction of $H_{n, c o n v, i n}$ and $H_{s o l, f i l m}, T_{f}$ also decreases until the sunset. As $T_{f}$ decreases, the temperature difference between $T_{a i r, o u t}$ and $T_{f}$ increases, and the ambient natural convection $H_{n, c o n v, o u t}$ increases. During nighttime, the thermal processes mainly effective to the film are $H_{I R l o s s, \text { Silm }}, H_{I R i n p u t, f i l m}$, and $H_{n, c o n v, \text { out }}$. Hence, during nighttime, $T_{f}$ is greater than the so-called "radiation equilibrium temperature" which is calculated only from the relation between $H_{\text {IRloss, film }}$ and $H_{\text {IRinput,film }}$. The ratio $T_{g} / T_{a i r, o u \iota}$ becomes minimum after the last ballasting, and the minimum value is smaller than that in Stage 1, because the temperature difference between $T_{a i r, \text { out }}$ and $T_{g}$ is larger. After the last ballasting, the free lift $f$ and the velocity become nearly equal zero.

After sunrise at the balloon altitude, $H_{s o l, g a s}$ and $H_{\text {sol,film }}$ begin to increase, $T_{g}$ and $T_{f}$ increase, $f$ becomes positive, and the balloon starts ascending. The large ascending velocity causes the large adiabatic cooling and the large forced convection. Because of the increases of both the forced convection and $H_{s o l, f i l m}, T_{f}$ increases, while the increase of the adiabatic cooling suppresses the increase of $T_{g}$. Hence $T_{f}$ is larger than $T_{g}$ almost throughout the ascending period, and the thermal energy flow of the interior convection is directed from the film to the gas.

Compared with the Stage 1, the balloon reaches a ceiling height earlier, because its ascending velocity is larger. Several times of hunting phenomena occur until the next noon, but the expelled amount is largest at the first time. Because of the large expulsion, the balloon suddenly stops, and both the adiabatic cooling and the forced convection are suddenly diminished. Then $T_{g}$ steeply changes to a higher value and becomes greater than $T_{f}$. After the first time expulsion, features are similar to the Stage 1. It is noted that the first expulsion occurs before the sunrise at the ground level (before $H_{\text {albedo,gas }}$ starts to increase) and the second one occurs after that.

\subsubsection{Stage 3: no ballasting with sunset}

Figure 11 shows an example of the data in Stage 3, from the 17th day noon until the 18th day noon. Before the solar zenith angle is approaching $90^{\circ}$, features are similar to the Stages 1 and 2 . As $H_{\text {sol,gas }}$ steeply decreases until sunset, $T_{g}$ decreases, $f$ changes to a large negative value, and the descending velocity increases. Since there are no ballastings, the balloon continues to descend below the controlled height. As the altitude decreases, $T_{a i r, o u t}$ decreases. The large descending velocity causes the large adiabatic heating which suppresses the reduction of $T_{g}$. Hence, the ratio $T_{g} / T_{\text {air,out }}$ is almost unchanged.

During nighttime, the balloon continues to descend, and the adiabatic heating also continues. Since $H_{\text {sol,gas }}$ and $H_{\text {albedo,gas }}$ become zero, the adiabatic heating suppresses the reduction of $T_{g}$, and the descending motion is gradually decelerated. Because the decrease of $T_{\text {air,out }}$ becomes slow, the ratio $T_{g} / T_{a i r, \text { out }}$ is almost unchanged. Because of the adiabatic heating, $T_{g}$ is greater than $T_{f}$ even during nighttime. 
Stage 2 : ballasting with sunset

(a)

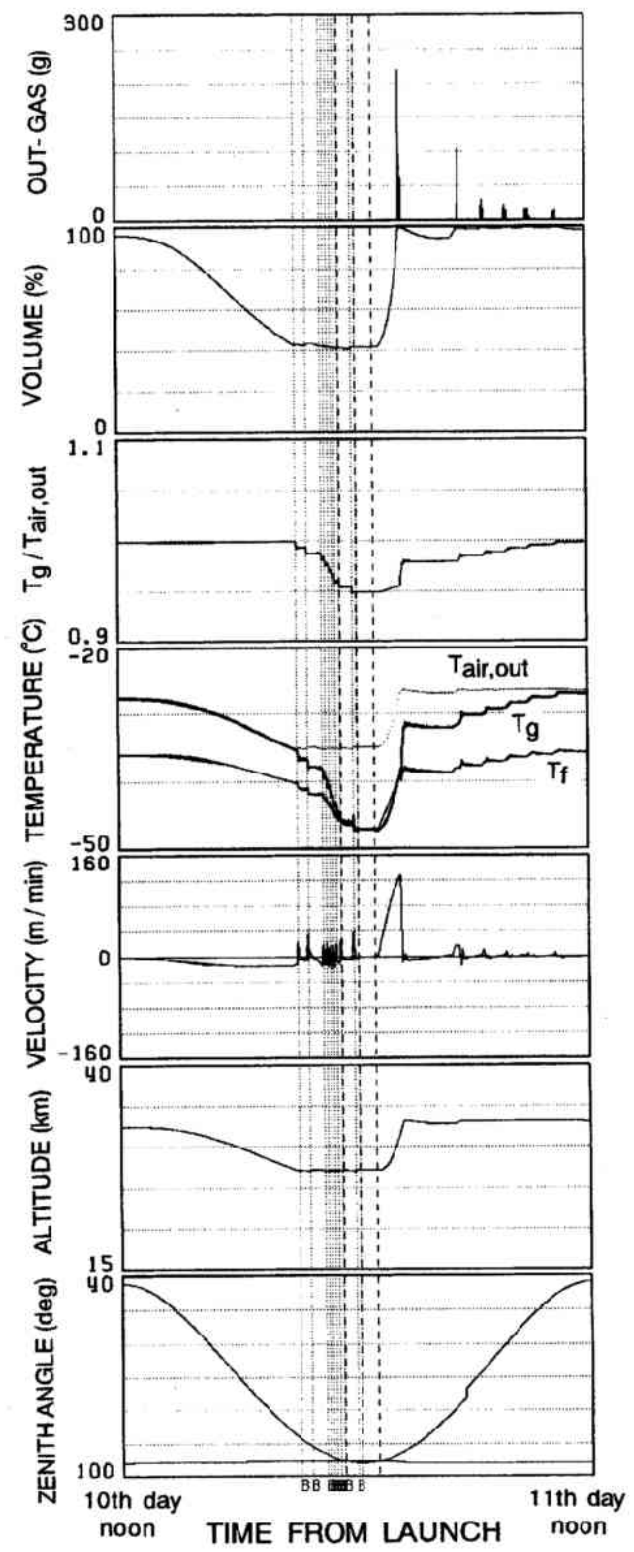

(b)

CONTRIBUTION OF EACH THERMAL PROCESS
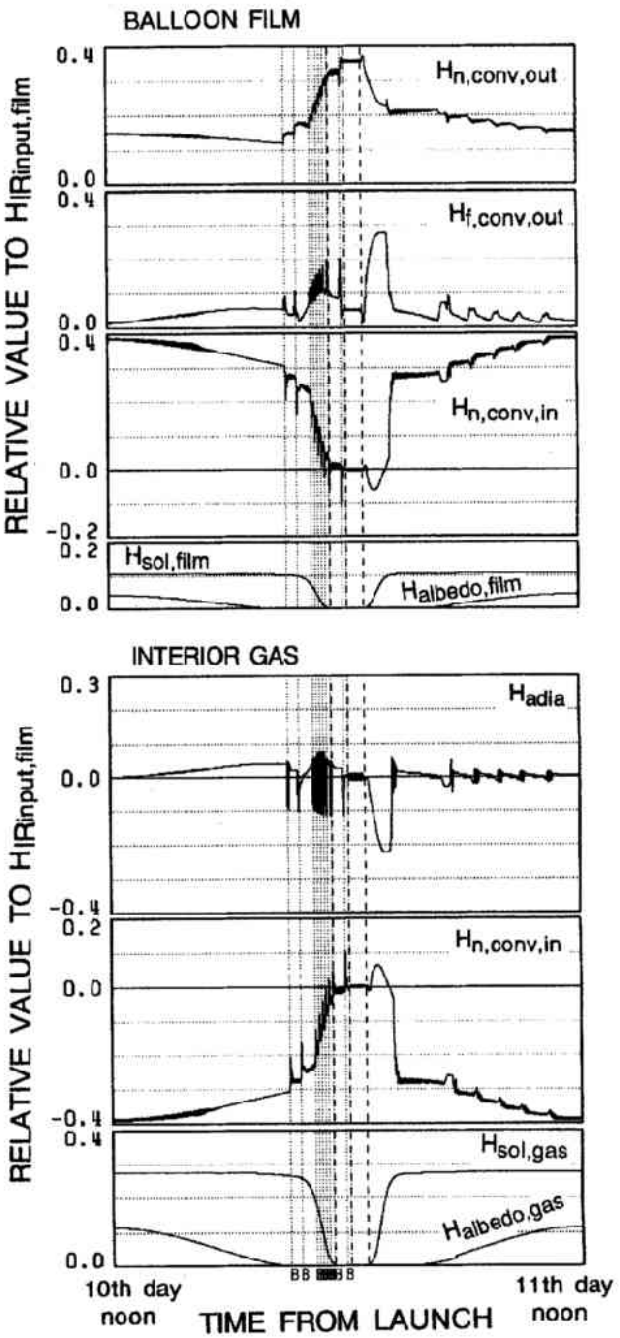

Fig. 10. An example of the data in Stage 2. Format is same as Fig. 9. Both abscissas of (a) and (b) represent time, from the 10 th day noon until the 11 th day noon. Two vertical break lines show sunset and sunrise at the balloon position. 
Stage 3 : no ballasting with sunset

(a)

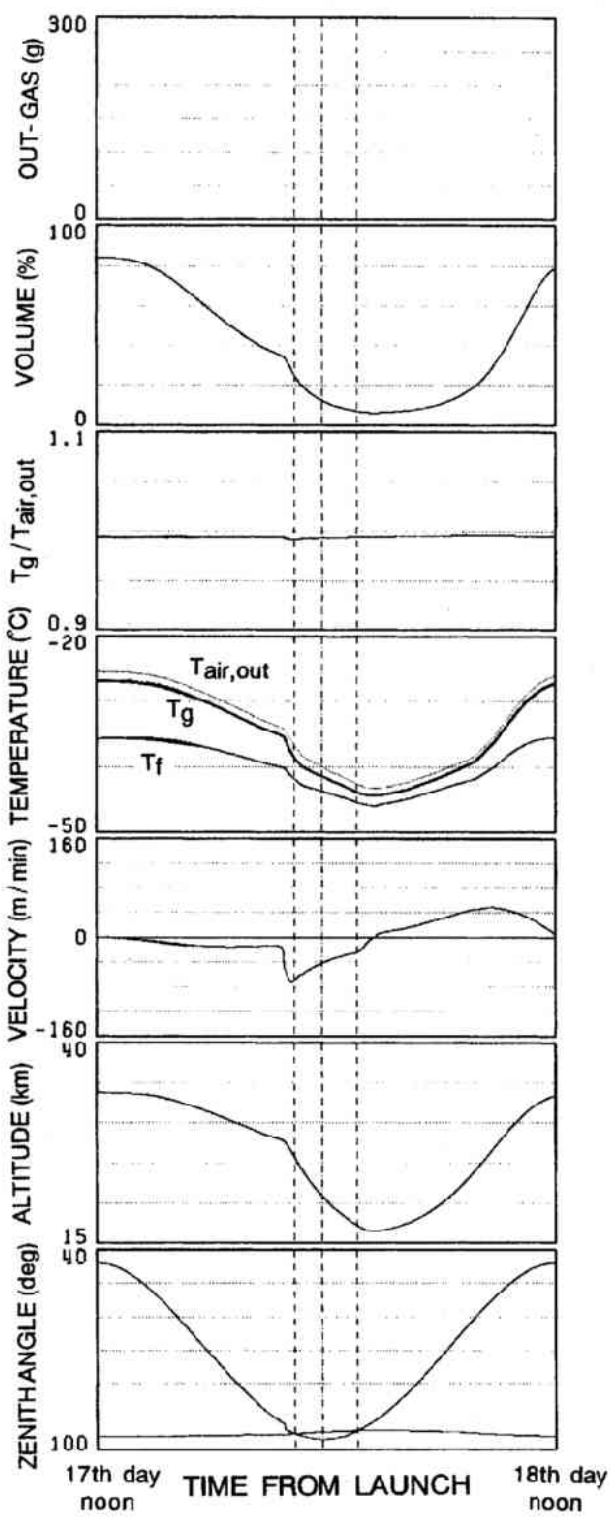

(b)

CONTRIBUTION OF EACH THERMAL PROCESS
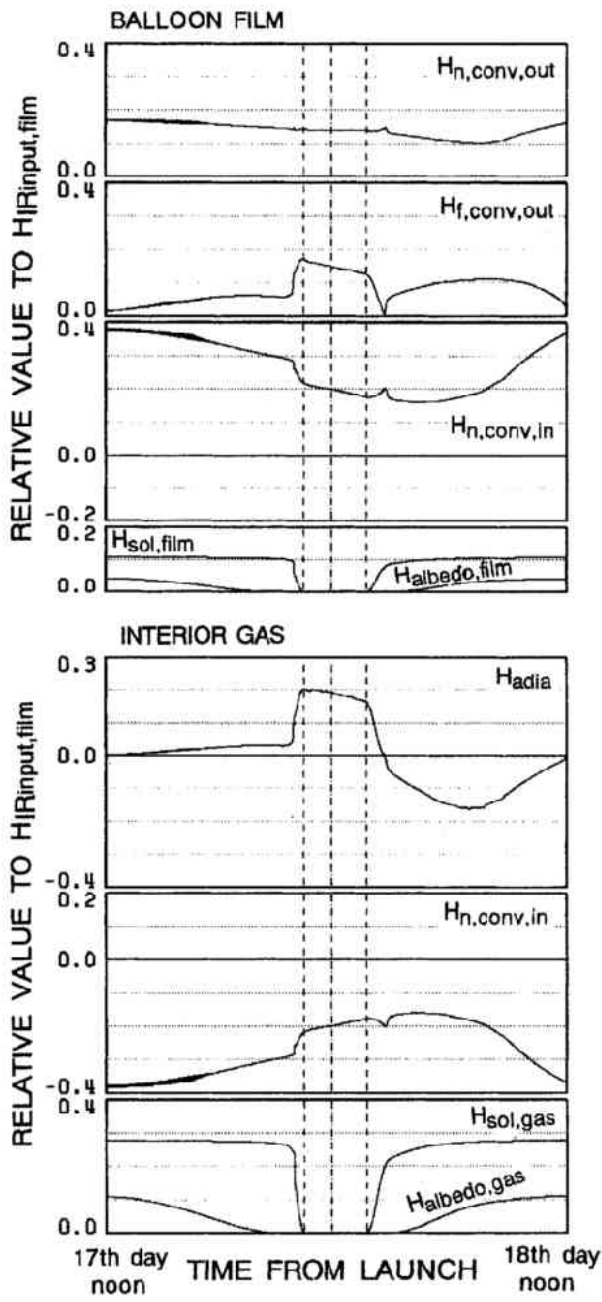

Fig. 11. An example of the data in Stage 3. Format is same as Fig. 10. Both abscissas of (a) and (b) represent time, from the 17 th day noon until the 18 th day noon. 
As $H_{\text {sol,gas }}$ steeply increases after sunrise, the decrease of $T_{g}$ is much slowed, and the descending motion is strongly decelerated. It is always after sunrise that the balloon reaches a minimum altitude and starts ascending. Hence $T_{g}$ at the minimum altitude is not the "radiation equilibrium temperature" calculated only from the relation between $H_{\text {IRloss,film }}$ and $H_{\text {IRinput,film }}$ as frequently assumed in previous works (e.g. Lally, 1983). After the sunrise at the ground level, $H_{\text {albedo,gas }}$ increases, and both the ascending velocity and the adiabatic cooling increase. When the increase of $H_{\text {albedo,gas }}$ is slowed, these two values decrease, and the increase of $T_{g}$ becomes large. Even at the next noon the balloon continues to ascend, and it is always after noon that the balloon reaches a maximum altitude. The ratio $T_{g} / T_{\text {air,out }}$ is almost unchanged during the ascending period. The ratio at the next noon is a little higher than that at the previous noon. Throughout this stage, the balloon volume never becomes maximum, and no expulsions occur.

Figure 12 shows the time variation of the relation between $T_{g}$ and $T_{a i r, \text { out }}$ in each Stage mentioned above. The feature in Stage 3 is apparently different from the other Stages. In Stages 1 and 2, the period from "B" to "C" corresponds to the ballasting period when $T_{\text {air,out }}$ is maintained around $\left.T_{\text {air,out }}\right|_{B}$, and the period from " $\mathrm{D}$ " to " $\mathrm{E}$ " does the gas expulsion period when $T_{\text {air,out }}$ becomes a nearly constant value around the maximum altitude $\left.T_{\text {air,out }}\right|_{\text {max }}$. The other periods from " $\mathrm{A}$ " to " $\mathrm{B}$ " and from " $\mathrm{C}$ " to " $\mathrm{D}$ " correspond to the descending and the ascending periods, respectively. $\left.T_{g}\right|_{\min }\left(T_{g}\right.$ at "C") is smaller and $\left.T_{\text {air,out }}\right|_{\text {max }}$ is larger in Stage 2 than in Stage 1. Since the ratio $T_{g} / T_{a i r, o u t}$ is almost unchanged during both the descending and ascending periods, the time variations from " $\mathrm{A}$ " to " $\mathrm{B}$ " and from " $\mathrm{C}$ " to " $\mathrm{D}$ " are approximately indicated by two straight lines in Figs. 12(a) and (b), and the gradient for the descending period is always larger than for the ascending one.

In Stage 3, since there are no ballastings and no gas expulsions, there exist only two separated periods, namely the descending period (from "A" to "B") and the ascending one (from " $\mathrm{C}$ " to " $\mathrm{D}$ "). In this Stage, points "B" and "C" are coincided with each other. Time variations during these periods are approximately indicated by two straight lines in Fig. 12(c), and the gradient for the descending period is a little smaller than for the ascending one. Time advances clockwise in Stage 3, while counter-clockwise in the other Stages.

Time histories of the ratio $T_{g} / T_{a i r, \text { out }}$ at noon and at midnight are shown in Fig. 7 (b).

(a) Stage 1

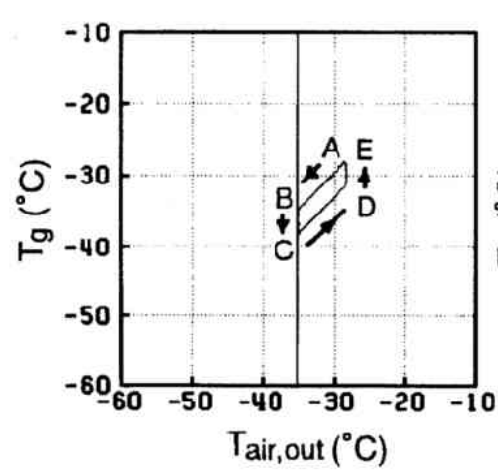

(b) Stage 2

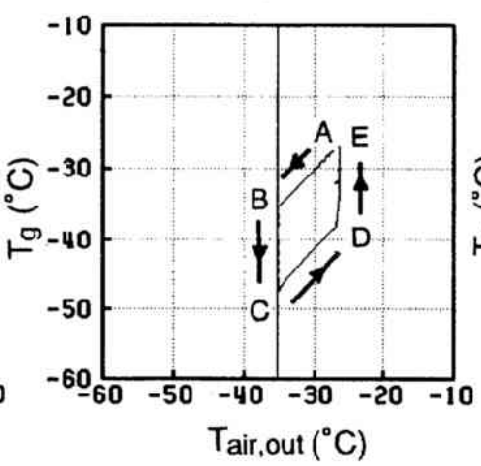

(c) Stage 3

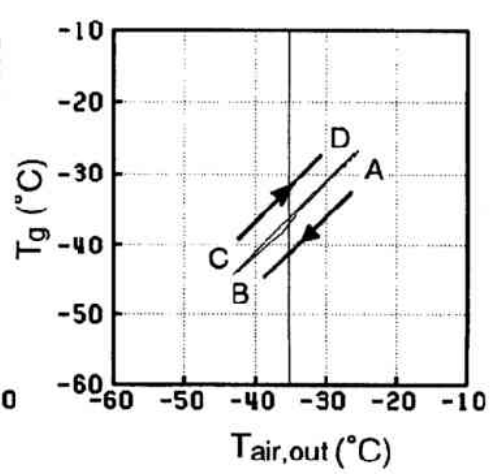

Fig. 12. Time variations of the relation between $T_{g}$ and $T_{\text {air, out }}$ in the three different Stages. (a), (b), and (c) correspond to the Stages 1, 2, and 3, respectively. Time advances in each panel as shown by arrows from point "A" (previous noon) to "E" or "D" (next noon). Periods of (a), (b), and (c) are the same with those in Fig. 9, Fig. 10, and Fig. 11, respectively. The vertical line around $-35^{\circ} \mathrm{C}$ of $T_{\text {air,out }}$ indicates the atmospheric temperature at the controlled height. 
The noon value gradually decreases in Stages 1 and 2 because the maximum altitude of the balloon increases ( $T_{\text {air,out }}$ increases) day by day, and in Stage 3 it gradually increases because the altitude decreases. Regarding the midnight, $T_{\text {air,out }}$ keeps the almost constant value at the controlled height in Stages 1 and 2. The ratio $T_{g} / T_{\text {air,out }}$ sharply decreases in Stage 1 because the midnight value of $T_{g}\left(\left.T_{g}\right|_{\text {midnight }}\right)$ sharply decreases as the balloon approaches the area where sunset exists. The ratio becomes almost constant in Stage 2 because the balloon enters the area and $\left.T_{g}\right|_{\text {midnight }}$ becomes almost constant. In Stage 3, it becomes nearly equal to, but a little smaller than the noon value, and gradually increases because the balloon altitude at midnight gradually decreases.

\section{Discussions}

\subsection{Dynamics in the three Stages}

Variation of the free lift determines the vertical motion of the calculated balloon, which is expressed as follows from Eq. (8):

$$
\Delta f=\Delta m_{\text {gas }}\left(\frac{M_{\text {air }}}{M_{\text {gas }}} \frac{T_{g}}{T_{\text {air }, \text { out }}}-1\right) g+m_{\text {gas }} \frac{M_{\text {air }}}{M_{\text {gas }}} \Delta\left(\frac{T_{g}}{T_{\text {air }, \text { out }}}\right) g-\Delta M g .
$$

$\triangle f$ is very small throughout the flight, except just after the ballastings or expulsions occur.

\subsubsection{Stages 1 and 2}

(a) Descending period ( $A \rightarrow B$ in Fig. 12)

In this period, $\Delta M=0$ because no ballastings occur, and $\Delta m_{\text {gas }}$ is a very small negative value because no expulsions occur and only the diffusion loss process is operated. Hence, Eq. (9) can be approximately written as $\Delta f \approx m_{\text {gass }} M_{\text {air }} / M_{\text {gass }} \Delta\left(T_{g} / T_{\text {air }, \text { out }}\right) g$. Both $\triangle f$ and $\triangle\left(T_{g} / T_{\text {air, out }}\right)$ are very small, hence $T_{g} / T_{a i r, \text { out }}$ is almost constant during the descending period, which means that

$$
\left(\frac{T_{g}}{T_{\text {air }, \text { out }}}\right)_{B} \approx\left(\frac{T_{g}}{T_{\text {air,out }}}\right)_{A} .
$$

(b) Ballasting period $(B \rightarrow C$ )

In this period, $\Delta m_{\text {gas }}$ is again negligibly small, and $\Delta f$ is also very small except just after the ballastings occur. Hence, the following relation can be written from Eq. (9):

$$
\Delta M \approx m_{\text {gas }} \frac{M_{\text {air }}}{M_{\text {gas }}} \Delta\left(\frac{T_{g}}{T_{\text {air }, \text { out }}}\right) .
$$

Since $T_{a i r, o u t}$ is almost constant at the controlled height, $T_{g}$ decreases at each ballasting. If Eq. (11) is integrated from the time B until the time C, total daily ballasting amount $-\Delta M_{\text {total }}$ is derived as

$$
-\Delta M_{\text {total }} \approx m_{\text {gas }} \frac{M_{\text {air }}}{M_{\text {gas }}}\left\{\left(\frac{T_{g}}{T_{\text {air }, \text { out }}}\right)_{B}-\left(\frac{T_{g}}{T_{a i r, o u t}}\right)_{C}\right\} .
$$

Since $m_{\text {gas }}$ is almost constant from A to C, Eq. (12) can be written using Eq. (10) as follows:

$$
\begin{aligned}
-\Delta M_{\text {total }} & \approx\left(m_{\text {gas }}\right)_{A} \frac{M_{\text {air }}}{M_{\text {gas }}}\left\{\left(\frac{T_{g}}{T_{\text {air }, \text { out }}}\right)_{A}-\left(\frac{T_{g}}{T_{\text {air }, \text { out }}}\right)_{C}\right\} \\
& \approx\left(m_{\text {gas }}\right)_{\text {noon }} \frac{M_{\text {air }}}{M_{\text {gas }}}\left\{\left(\frac{T_{g}}{T_{\text {air }, \text { out }}}\right)_{\text {noon }}-\left(\frac{T_{g}}{T_{\text {air }, \text { out }}}\right)_{\text {midnight }}\right\} .
\end{aligned}
$$

As shown in Fig. 13, $-\triangle M_{\text {total }}$ calculated from Eq. (13) is consistent very well with the calculated daily ballasting amount. The largest difference between them is $1.2 \mathrm{~kg}$, which is about the ballasting unit, $1 \mathrm{~kg}$, in this model. Hence, the ballasting process can be analyzed with Eq. (13). 


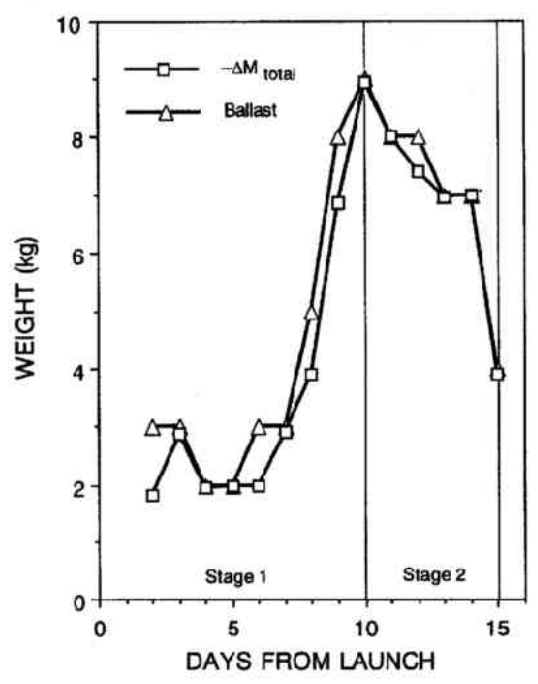

Fig. 13. $-\triangle M_{\text {total }}$ calculated by Eq. (13) (squares), compared with the ballasting amount (triangles) in the model calculation.

In Stage 1, the ballasting amount increases as the balloon approaches the sunset area because the noon-midnight difference of $T_{g} / T_{\text {air, out }}$ sharply increases as shown in Fig. 7 (b). In Stage 2 when the balloon enters the sunset area, the noon-midnight difference of the temperature ratio becomes almost constant, and the ballasting amount decreases because $\left(m_{\text {gas }}\right)_{\text {noon }}$ decreases mainly due to the expulsion process. It should be noted that duration of sunset does not appear in Eq. (13) and hence has no explicit relation with the ballasting amount.

If $\left(T_{g} / T_{\text {air,out }}\right)_{\text {midnight }}$ increases in Eq. (13), the daily ballasting amount decreases. $\left(T_{\text {air,out }}\right)_{\text {midnight }}$ is nearly equal the value at the controlled height $\left(T_{\text {air,out }}\right)_{B}$. For a fixed value of $\left(T_{g}\right)_{\text {midnight }}$, if $\left(T_{a i r, o u t}\right)_{B}$ is set to a smaller value (if the controlled height is set at a lower altitude in the stratosphere), the ballasting amount decreases. In an extreme case in which $\left(T_{a i r, o u t}\right)_{B}$ is smaller than $\left(T_{\text {air,out }} / T_{g}\right)_{\text {noon }}\left(T_{g}\right)_{\text {midnight }}\left(\equiv\left(T_{\text {air,out }}\right)_{\text {midnight }}\right)$, no ballastings occur, and the balloon moves vertically in a wide altitude range between $\left(T_{\text {air,out }}\right)_{\text {midnight }}$ and $\left(T_{\text {air,out }}\right)_{\text {noon }}$, which is the concept of RACOON introduced in Lally (1983) and the similar situation to Stage 3 in this calculation. Another way to reduce the ballasting amount is to make $\left(T_{g} / T_{\text {air, out }}\right)_{\text {noon }}$ in Eq. (13) small. If $\left(T_{g}\right)_{\text {noon }}$ becomes small, the balloon volume becomes $V_{\text {full }}$ at noon at a higher altitude (lower pressure altitude) because of the zero-pressure balloon condition. Hence, $\left(T_{a i r, o u t}\right)_{n o o n}$ increases and $\left(T_{g} / T_{\text {air,out }}\right)_{\text {noon }}$ becomes small. It will be discussed about $\left(T_{g}\right)_{\text {midnight }}$ and $\left(T_{g}\right)_{\text {noon }}$ in Subsection 5.2.

(c) Ascending period $(C \rightarrow D)$

This period is similar to the descending one. $\Delta M=0, \Delta m_{g a s}$ and $\Delta f$ are very small, and $\triangle\left(T_{g} / T_{a i r, o u t}\right)$ is also very small. Hence $T_{g} / T_{a i r, \text { out }}$ is almost constant throughout this period and always smaller than in the descending period. When the balloon ascends and reaches the altitude at previous noon at the time $t_{0},\left(T_{\text {air,out }}\right)_{t_{0}}$ equals $\left(T_{\text {air,out }}\right)_{A}$ in this calculation. Since $\left(T_{g} / T_{\text {air,out }}\right)_{t_{0}}$ is smaller than $\left(T_{g} / T_{\text {air,out }}\right)_{A},\left(T_{g}\right)_{t_{0}}$ is smaller than $\left(T_{g}\right)_{A}$ and the balloon volume does not yet become maximum at the time $t_{0}$. Hence the balloon continues to ascend and becomes maximum volume always at a higher altitude than at the previous noon. Consequently the maximum altitude of the calculated balloon in Stages 1 and 2 increases day by day. 
(d) Expulsion period $(D \rightarrow E)$

In this period, $\triangle M=0$, and $\triangle f$ is very small except just after the expulsions occur. Hence, the following relation can be written from Eq. (9):

$$
\Delta\left(\frac{T_{g}}{T_{\text {air }, \text { out }}}\right)=-\frac{\Delta m_{\text {gas }}}{m_{\text {gas }}}\left(\frac{T_{g}}{T_{\text {air }, \text { out }}}-\frac{M_{\text {gas }}}{M_{\text {air }}}\right) .
$$

Since $T_{\text {air,out }}$ is almost constant at the ceiling height, $T_{g}$ increases at each expulsion.

\subsubsection{Stage 3}

In this Stage, $\triangle M=0$ and $\triangle f$ is very small throughout every period. Hence the same relation with Eq. (14) describes the balloon motion. Since the interior gas weight gradually decreases by diffusion, $T_{g} / T_{a i r, \text { out }}$ gradually increases from Eq. (14). For a fixed $T_{g}, T_{a i r, \text { out }}$ gradually decreases, which means that the calculated balloon altitudes gradually decreases. If the cross section of the venting duct is smaller, the diffusion loss becomes smaller and the decreasing rate of the balloon altitude becomes smaller. Since $\Delta m_{\text {gas }}$ is very small, $\Delta\left(T_{g} / T_{\text {air,out }}\right)$ is also very small, and $T_{g} / T_{\text {air,out }}$ is almost constant throughout the descending and ascending periods in this Stage.

\subsection{Thermal equilibrium temperatures}

Except just after the ballastings or expulsions occur, thermal condition of the balloon is almost in an equilibrium state throughout the flight, which can be seen in Figs. 9(b), 10(b), and 11(b). If $d T_{f} / d t$ and $d T_{g} / d t$ are set to zero and minor terms, $H_{I R i n t}, H_{\text {IRloss,gas }}, H_{\text {IRinput,gas }}$ are neglected in Eqs. (2) and (3), then following equation can be derived by adding Eqs. (2) and (3):

$$
\begin{aligned}
T_{f}=\left[\frac { 1 } { 4 \sigma } \left\{\frac{\varepsilon_{\text {eff }}+\varepsilon_{g, \text { eff }}}{\kappa_{\text {eff }}}\left(A_{p}+A_{A}\right) J_{0}\right.\right. \\
\left.\left.+2(1+e) J_{1}+\frac{H_{n, \text { conv }, \text { out }}+H_{f, \text { conv }, \text { out }}+H_{\text {adia }}}{\pi r_{B} \kappa_{\text {eff }}}\right\}\right]^{1 / 4}
\end{aligned}
$$

and from Eq. (3),

$$
T_{g}=T_{f}+\frac{r_{B}}{2 a_{1} K_{g a s} N u_{n, i n}}\left\{\varepsilon_{g, e f f}\left(A_{p}+A_{A}\right) J_{0}+\frac{H_{a d i a}}{\pi r_{B}^{2}}\right\} .
$$

(a) Values at noon

At noon, both $H_{f, c o n v, \text { out }}$ and $H_{\text {adia }}$ are nearly equal zero. Since $\varepsilon_{g, e f f}\left(\approx \varepsilon_{g}\right)$ is a finite value in this model, $\left(T_{g}\right)_{n o o n}$ is always larger than $\left(T_{f}\right)_{n o o n}$ from Eq. (16). The value of $\varepsilon_{g}$ is one of the most effective parameter in this model to the daily ballasting amount and the altitude variation. As $\varepsilon_{g}$ becomes larger, $\left(T_{f}\right)_{n o o n},\left(T_{g}\right)_{n o o n}$, and difference between them become larger, and daily ballasting amount increases as discussed in Subsection 5.1.1(b). If $\varepsilon_{g}$ is set to zero, which is the case in Nishimura et al. (1973) or Lally $(1983),\left(T_{g}\right)_{n o o n}$ becomes small and nearly equals to $\left(T_{f}\right)_{\text {noon }}$. In a test calculation with such condition, there are no ballastings occur throughout the calculation period. It is found that $\varepsilon_{g}=0.0052$ gives a most consistent result with the observation concerning the daily ballasting amount. As mentioned in Subsection 3.2, radiative properties $\varepsilon_{\text {eff }}, \kappa_{\text {eff }}$ are also very important factors affecting the balloon motion. To make $\left(T_{g}\right)_{\text {noon }}$ small, $\kappa_{\text {eff }}$ should become larger, and $\varepsilon_{\text {eff }}$ become smaller. In other words, one possible way to reduce the ballasting amount is to use such a material for the balloon film whose IR emissivity/absorptivity $\kappa_{0}$ is large and solar absorptivity $\varepsilon_{0}$ is small. From Eqs. (15) and (16), it can be seen that the albedo of the earth is also effective to the value of $\left(T_{g}\right)_{\text {noon }}$. If the balloon floats above a high-albedo (low-albedo) area, $\left(T_{g}\right)_{\text {noon }}$ increases (decreases), and hence the daily 
ballasting amount increases (decreases). Since there is a sufficient temperature difference between the ambient air and the balloon film, $H_{n, c o n v, o u t}$ in Eq. (15) is not negligible.

\section{(b) Values at midnight}

In Stages 1 and $2, H_{f, c o n v, o u t}$ and $H_{\text {adia }}$ are nearly equal zero. Both $A_{p}$ and $A_{A}$ decrease as the balloon approaches the sunset area in Stage 1, and become zero in Stage 2. Hence $\left(T_{g}\right)_{\text {midnight }}$ is nearly equal to $\left(T_{f}\right)_{\text {midnight }}$ in Stage 2. $H_{n, \text { conv,out }}$ in Eq. (15) is not negligible because the temperature difference between the ambient air and the balloon film is sufficiently large, especially in Stage 2. In Stage 3, the balloon continues to descend even at midnight. Hence $H_{f, c o n v, o u t}$, $H_{\text {adia }}$, and $H_{n, \text { conv,out }}$ are not negligible in Eqs. (15) and (16). $\left(T_{g}\right)_{\text {midnight }}$ is always greater than $\left(T_{f}\right)_{\text {midnight }}$ because of the adiabatic heating. In all the three Stages, $\left(T_{g}\right)_{\text {midnight }}$ is always greater than the so-called "radiation equilibrium temperature", as mentioned in Subsection 4.2.

\subsection{Difference between the observation and the calculation}

(a) Ballasting amount

In the calculation, ballasting starts from the first day, i.e. 2 days earlier, and the ballasting amount becomes maximum on the 10th day, i.e. 1 day earlier than in the observation. As discussed in Subsection 5.1.1, the maximum day should correspond to the day when sunset first appears at the balloon position. If this is true even in the actual experiment, the sunset in the actual case should first appear on the 11th day. However, from geometrical calculation, sunset does first appear on the 10th day even in the actual case as discussed in Kadokura et al. (1991). One possible explanation to this discrepancy is that there was twilight effect after the geometrical sunset, and effective solar radiation came to the balloon even on the 10th day, and this effect disappeared on the 11th day. The late start of the ballasting in the observation can be also explained by this twilight effect. The twilight region on the ground enlarges the albedo area on the ground, which means that $A_{A}$ becomes larger especially at midnight. Consequently, $\left(T_{g}\right)_{\text {midnight }}$ increases from Eqs. (15) and (16), and the ballasting may not occur during the first two days in the actual experiment, as discussed in Subsection 5.1.1. For further consideration, this twilight effect should be included in the present model.

If the albedo of the earth surface in the actual case is smaller (larger) than the simplified value in the calculation, $\left(T_{g}\right)_{\text {noon }}$ becomes smaller (larger) and the ballasting amount decreases (increases). Such a spatial and temporal variation of the albedo may also cause the discrepancy between the observation and the calculation concerning the ballasting amount.

Altitude profile of the air temperature in the actual case also varies spatially and temporarily. If $\left(T_{\text {air,out }}\right)_{\text {noon }}$ becomes smaller (larger) or $\left(T_{\text {air, out }}\right)_{\text {midnight }}$ becomes larger (smaller), the ballasting amount increases (decreases) from Eq. (13), which may be an explanation to the discrepancy concerning the ballasting amount. Different altitude profile of the air temperature shown by a break line in Fig. 3 was tested, and it is found that the daily ballasting amount becomes larger than for the profile shown by a straight line in Fig. 3, and the total ballast has been dropped on the 13th day. Even in this case, the ballasting amount reaches a maximum on the 10th day.

(b) Vertical velocity

Both upward and downward velocities in the calculation are larger than those in the observation. In a nearly steady state condition, the vertical velocity $v_{\perp}$ is expressed from Eq. (1) as follows;

$$
v_{\perp} \approx \sqrt{\frac{2 f}{C_{0} \rho_{a i r} S}} .
$$

$v_{\perp}$ increases if the air drag coefficient $C_{0}$ decreases. In this model, $C_{0}$ is calculated from the Reynolds number using the laboratory data for a sphere. The actual shape of the balloon is not sphere and changes depending on the balloon volume. Throughout the flight, the Reynolds 
number is almost between $10^{1}$ and $10^{5}$. Hence, it may be said that $C_{0}$ for the actual balloon should be larger than for a sphere, at least in the range of the Reynolds number, from $10^{1}$ to $10^{5}$. Another explanation to this discrepancy is such that the eddy viscosity caused by the natural turbulent flow in the stratosphere is much larger than the molecular viscosity treated in the laboratory data, hence the Reynolds number becomes smaller and $C_{0}$ becomes larger in the actual case.

(c) Altitude change

After the auto-ballast control ceased functioning, the amplitude of the altitude change during one day is larger in the calculation than in the observation, because both upward and downward velocities in the calculation are larger. While the auto-ballast control is operated, the balloon in the calculation stays around the maximum altitude until next noon, while the actual balloon started descending soon after it reached the maximum altitude. Hence, only one time of gas expulsion should occur in the actual case, while several times of expulsion occur in the calculation. If the total amount of expulsion during one day is same both in the actual case and in the calculation, the amount of the expulsion at the one time in the actual case should be very large. In other words, the amount of the first time expulsion in the actual case is so large that the free lift changes to a large negative value and the balloon cannot ascend again. Such a large amount of expulsion should be caused by a large difference of the pressure between the interior and the ambient. If an effective expulsion could not occur until the pressure difference becomes sufficiently large, the amount of the first expulsion should be larger than in the present calculation. For further understanding, it is necessary to investigate the expulsion process more closely. It is known that there is a vertical flow in the stratosphere which is modulated by gravity wave. If the modulated vertical flow amplifies the hunting motion of the balloon, it may increase the expulsion amount. This may be another explanation to this discrepancy.

\subsection{Application of this model}

Present model can be used to simulate the vertical motions of any PPBs with various balloon volumes, payload weights, and auto-ballasting parameters (controlled height, dropping unit and interval, and total ballast payload). This model also can be applied to the analysis of the motions of the other kind of balloon experiments. For example, if the dropping unit is set to zero, there are no ballastings, and the model balloon simulates the zero-pressure balloon without ballastings, i.e. RACOON introduced in Lally (1983). If the cross section of the venting duct is set to zero, there are no gas expulsions, and the model balloon becomes a super-pressure balloon. As mentioned in Section 3.4, if the objective analysis data of Japan Meteorological Agency should be included in this model, this model can simulate not only the vertical motion but also the horizontal trajectory and can be used for prediction of future experiments using a conventional PPB, RACOON, or super-pressure balloon launched at various sites in various seasons for different purposes.

\section{Summary}

A numerical model to investigate the vertical motion of a PPB has been developed. This model was applied to the data of the JARE-30 PPB experiment, and could give a calculation result well consistent with the observation. The thermal and dynamic behaviors of the JARE30 PPB were analyzed by using this model calculation, and basic features of PPB system were elucidated.

There are following three different stages in the JARE-30 PPB experiment; Stage 1: ballasting without sunset; Stage 2: ballasting with sunset; Stage 3: no ballasting with sunset. In Stages 1 and 2, there are four distinct periods during one day, i.e. post-noon descending, night ballasting, morning ascending, and pre-noon expulsion. Every ballasting occurs before midnight and every expulsion occurs before noon. Daily ballasting amount is nearly proportional to the 
interior gas weight $m_{\text {gas }}$ and the noon-midnight difference of the temperature ratio of the interior gas to the ambient air $\triangle\left(T_{g} / T_{a i r, o u t}\right)$. As the balloon approaches the latitude region where sunset exists, $\triangle\left(T_{g} / T_{\text {air, out }}\right)$ increases and the ballasting amount increases. After the balloon enter the region, the ballasting amount decreases because $\triangle\left(T_{g} / T_{a i r, o u t}\right)$ becomes almost constant and $m_{\text {gas }}$ decreases mainly due to the expulsion process. Hence the day of the maximum ballasting amount corresponds to the day when the sunset time first appears at the balloon altitude, and sunset duration itself does not have any explicit relation with the ballasting amount. The ratio $T_{g} / T_{\text {air,out }}$ is almost constant during both the descending and ascending periods, and changes stepwise to the lower (higher) value at each ballasting (expulsion) due to the adiabatic cooling (heating) during the ballasting (expulsion) period. Since the ratio during the ascending period is smaller than during the descending one, the balloon does not become maximum volume when it ascends and reaches the same altitude at the previous noon. Hence the balloon continues to ascend and becomes maximum volume always at a higher altitude than at the previous noon. Consequently, the maximum altitude of the balloon in Stages 1 and 2 increases day by day.

In Stage 3, there are only descending and ascending periods because no ballastings and no expulsions occur. Since the interior gas weight continues to decrease by the diffusion process, the balloon altitude gradually decreases day by day.

In daytime, the interior gas temperature $T_{g}$ is always larger than the film temperature $T_{f}$ because the interior gas absorbs the direct and albedo solar radiations. In nighttime in Stages 1 and 2, there is a sufficient temperature difference between $T_{f}$ and the ambient air temperature $T_{a i r, o u t}$ because $T_{a i r, o u t}$ is confined to the value around the controlled height. Hence the contribution of the ambient natural convection to the thermal balance of the PPB system is not negligible. In Stage 2, nighttime value of $T_{g}$ becomes nearly equal $T_{f}$. In Stage 3 , the balloon continues to descend even in nighttime, and $T_{g}$ is always larger than $T_{f}$ because of the adiabatic heating. The contributions of the ambient natural and forced convections are not negligible in nighttime in this stage.

There are some discrepancies between the model calculation and the observation. In the calculation, ballasting starts 2 days earlier, and the ballasting amount becomes maximum 1 day earlier than in the observation. Twilight effect is a possible reason for this discrepancy and should be included in this model. Temporal and spatial variation of both the albedo and the altitude profile of the atmospheric parameters may also cause such a discrepancy. Vertical velocity in the calculation is larger than in the observation. One possible reason for this result is that the calculated drag coefficient is smaller than in the actual case. Further study should be made about the natural atmospheric turbulence and the drag coefficient for the actual shape of the balloon. The actual balloon started descending soon after it reached the maximum altitude, while the calculated balloon stays around the maximum altitude until next noon. One possible reason for this difference is that gas expulsion process in the actual case is different from in the calculation. It is necessary to investigate the expulsion process more closely.

In conclusion, the vertical excursion behaviors of the PPB system have been well understood and predicted by the present model. If more realistic atmospheric parameters are included in this model, it permits a prediction of not only the vertical motion but also the horizontal trajectory of the PPB, and can contribute to the scientific planning in the future project of the solar-terrestrial physics.

The author is very grateful to Prof. J. Nishimura and Dr. T. Yamagami for their useful comments and valuable suggestions, and to Dr. F. T. Berkey for his kindness to share his time to check the draft of this paper. He would like to thank all members of the Upper Atmosphere Physics group of NIPR for their generous supports to this kind of work. The PPB project have been done under the PPB Working Group (Chairman: Prof. N. Yajima). The author is very grateful to all members of the Working Group. He also 
wishes to express his cordial thanks to all members of JARE-30 led by Prof. M. Ejiri. This calculation was done with the M-680D computer of Information Science Center of NIPR. Special thanks are due to Messrs. H. Sakurai and K. Uchida who kindly instructed the author to handle the computer.

\section{Appendix: Details of the Parameters Used in the Model Equations}

The effective IR emissivity $\kappa_{\text {eff }}\left(\kappa_{g, e f f}\right)$ and the effective solar absorptivity $\varepsilon_{e f f}\left(\varepsilon_{g, e f f}\right)$ of the film (gas), and the effective IR emissivity for the interchange process between the gas and the film $\kappa_{\text {int }}$ are written as follows:

$$
\begin{aligned}
\kappa_{e f f} & =\kappa_{0}\left\{1+\frac{\tau_{I R}\left(1-\kappa_{g}\right)}{1-r_{I R}\left(1-\kappa_{g}\right)}\right\}, \\
\varepsilon_{\text {eff }} & =\varepsilon_{0}\left\{1+\frac{\tau_{s o l}\left(1-\varepsilon_{g}\right)}{1-r_{s o l}\left(1-\varepsilon_{g}\right)}\right\}, \\
\kappa_{g, \text { eff }} & =\frac{\kappa_{g} \tau_{I R}}{1-r_{I R}\left(1-\kappa_{g}\right)}, \\
\varepsilon_{g, \text { eff }} & =\frac{\varepsilon_{g} \tau_{s o l}}{1-r_{s o l}\left(1-\varepsilon_{g}\right)}, \\
\kappa_{\text {int }} & =\frac{\kappa_{g} \kappa_{0}}{1-r_{I R}\left(1-\kappa_{g}\right)}
\end{aligned}
$$

where $\kappa_{0}\left(\varepsilon_{0}\right), \tau_{I R}\left(\tau_{s o l}\right), r_{I R}\left(r_{s o l}\right)$ are the IR (solar) emissivity/absorptivity, transmissivity, and reflectivity of the film, respectively, and $\kappa_{g}\left(\varepsilon_{g}\right)$ is the IR (solar) emissivity/absorptivity of the interior gas. Values of these radiative properties used in this calculation are as follows:

$$
\begin{aligned}
\kappa_{0} & =0.031, \\
\tau_{I R} & =0.842, \\
r_{I R} & =0.127, \\
\varepsilon_{0} & =0.001, \\
\tau_{\text {sol }} & =0.885, \\
r_{\text {sol }} & =0.114, \\
\kappa_{g} & =0.169 \times\left(1.746 \times 10^{-6} T_{g}\right)^{0.8152}, \\
\varepsilon_{g} & =0.0052 .
\end{aligned}
$$

Radiative properties of the film are taken from Kiser (1980), and $\kappa_{g}$ is from Carlson and Horn (1981). As mentioned in Subsection 3.2, $\varepsilon_{g}$ is treated as an unknown parameter, and it is found that the above value gives the most consistent result with the observations.

The transmittance of the solar radiation $A_{p}$ is time-dependent and varies with the solar zenith angle $\alpha$ at the balloon position as follows:

$$
A_{p}=\left[\begin{array}{ll}
\exp \left(-k \frac{d_{B}}{h_{\infty}-h_{B}} P_{B}\right) & \left(\text { if } 0 \leq \alpha \leq \frac{\pi}{2}\right) \\
\exp ^{2}\left(-k_{\frac{d_{X}}{h_{\infty}-h_{X}}} P_{X}\right) / \exp \left(-k_{\frac{d_{\infty}}{h_{\infty}-h_{B}}} P_{B}\right) & \left(\text { if } \frac{\pi}{2}<\alpha \leq \alpha_{\text {sunset }}\right) \\
0 & \left(\text { if } \alpha_{\text {sunset }}<\alpha\right)
\end{array}\right.
$$

where $k=-\ln 0.82 / 1000$ (Nishimura, 1990). $h_{\infty}$ is the height at the top of atmosphere, and in this calculation $h_{\infty}$ is set to $100 \mathrm{~km}$. $P_{X}$ is the pressure at the tangential height $h_{X}$ from the balloon to the sun. $d_{B}$ and $d_{B^{\prime}}$ are the distances between the balloon and the top of atmosphere 
along the solar direction, and $d_{X}$ is between the tangential height and the top of atmosphere. $\alpha_{\text {sunset }}=\pi-\sin ^{-1}\left(R_{E} /\left(R_{E}+h_{B}\right)\right)$ is the geometrical sunset angle at the balloon position, where $R_{E}$ is the earth radius. The effect of twilight is not considered.

The albedo transmittance $A_{A}$ is calculated as

$$
A_{A}=\frac{A_{0}}{\pi} \int d S \frac{\cos \beta}{2\left(d_{G}-d_{B}\right)^{2}} \exp \left\{-k\left(\frac{d_{G_{0}}+d_{G}}{h_{\infty}} 1000-\frac{d_{B}}{h_{\infty}-h_{B}} P_{B}\right)\right\}
$$

where $A_{0}$ is the albedo of the ground surface; $d S$ is a unit area on the ground; $\beta$ is the solar zenith angle at the position; $d_{G_{0}}$ is the distance between the top of atmosphere and the unit area along the solar direction; $d_{G}$ is between the unit area and the top of atmosphere along the line toward the balloon; $d_{B}$ is between the balloon and the top of atmosphere along the line. The albedo radiation is assumed to be emitted uniformly into a solid half angle from each position. The integration in Eq. (A.8) is carried out within the area where both the zenith angle to the balloon $\zeta$ and $\beta$ are less than $90^{\circ} . A_{A}$ is time-dependent, depending on the solar zenith angle and the position of the balloon.

The coefficient representing the height dependence of the IR radiation from the earth $e$ is found to be nearly zero at heights above $100 \mathrm{mb}$ according to Nishimura et al. (1973). Because the altitude to be mainly considered is above $100 \mathrm{mb}, e$ is set to zero in this calculation.

The coefficients of effective surface for each convection $a_{1}, a_{2}, a_{3}$ are set to $0.5,1, \pi / 4$, respectively.

The thermal conductivities of the interior gas $K_{g a s}$ and the ambient air $K_{a i r}$ are expressed as follows:

$$
\begin{aligned}
& K_{\text {gas }}=\left\{0.007728 \times\left(T_{g}-273.15\right)+3.328\right\} \times 10^{-4} \quad(\mathrm{cal} / \mathrm{cm} \mathrm{K} \mathrm{s}), \\
& K_{a i r}=\left\{0.001899 \times\left(T_{\text {air }, \text { out }}-273.15\right)+0.5702\right\} \times 10^{-4} \quad(\mathrm{cal} / \mathrm{cm} \mathrm{K} \mathrm{s}) \text {. }
\end{aligned}
$$

The Nusselt numbers for each convection $N u_{n, i n}, N u_{n, o u t}, N u_{f, \text { out }}$ are expressed in the following forms, according to Carlson and Horn (1981),

$$
\begin{aligned}
N u_{n, \text { in }} & =\left[\begin{array}{ll}
2.5 \times\left\{2+0.6 \times\left(G r_{\text {gas }} \cdot P r_{\text {gas }}\right)^{\frac{1}{4}}\right\} & \left(\text { if } G r_{\text {gas }} \cdot P r_{\text {gas }}<1.5 \times 10^{8}\right) \\
0.325 \times\left(G r_{\text {gas }} \cdot P r_{\text {gas }}\right)^{\frac{1}{3}} & \left(\text { if } G r_{\text {gas }} \cdot P r_{\text {gas }} \geq 1.5 \times 10^{8}\right), \\
N u_{n, \text { out }} & =2+0.6 \times\left(G r_{a i r} \cdot P r_{a i r}\right)^{\frac{1}{4}},
\end{array}\right. \\
N u_{f, \text { out }} & =0.37 \times R e_{\text {air }} 0.6
\end{aligned}
$$

where $G r_{\text {gas }}\left(G r_{a i r}\right)$ and $P r_{\text {gas }}\left(P r_{a i r}\right)$ are Grashof number and Prandtl number for the interior gas (for the ambient air), respectively, which are expressed as follows:

$$
\begin{aligned}
G r_{\text {gas }} & =\frac{\rho_{\text {gas }}^{2} g e_{\text {gas }}\left(2 r_{B}\right)^{3}\left|T_{g}-T_{f}\right|}{\mu_{\text {gas }}{ }^{2}} \\
G r_{a i r} & =\frac{\rho_{a i r}{ }^{2} g e_{a i r}\left(2 r_{B}\right)^{3}\left|T_{a i r}-T_{f}\right|}{\mu_{a i r}{ }^{2}} \\
P r_{\text {gas }} & =\frac{C p_{g} \mu_{\text {gas }}}{K_{\text {gas }}} \\
\operatorname{Pr}_{\text {air }} & =\frac{C p_{\text {air }} \mu_{a i r}}{K_{a i r}}
\end{aligned}
$$

where $C p_{a i r}$ is the specific heat at constant pressure of the ambient air; $\rho_{\text {gas }}\left(\rho_{\text {air }}\right), e_{\text {gas }}\left(e_{\text {air }}\right)$, and $\mu_{\text {gas }}\left(\mu_{\text {air }}\right)$ are the density, coefficient of cubical expansion, and coefficient of viscosity of the 
interior gas (of the ambient air), respectively:

$$
\begin{aligned}
& e_{\text {gas }}=\quad 3.6580 \times 10^{-3} \quad(/ \mathrm{K}), \\
& e_{\text {air }}=\quad 3.6728 \times 10^{-3} \quad(/ \mathrm{K}), \\
& \mu_{\text {gas }}=\frac{T_{g}{ }^{1.5}}{T_{s}+30} \times 0.1249 \times 10^{-4} \quad(\mathrm{~g} / \mathrm{s} \mathrm{cm}) \text {, } \\
& \mu_{\text {air }}=\frac{T_{\text {air }}{ }^{1.5}}{T_{\text {air }}+117} \times 0.1479 \times 10^{-4}(\mathrm{~g} / \mathrm{s} \mathrm{cm}) .
\end{aligned}
$$

$R e_{a i r}$ is the Reynolds number:

$$
R e_{a i r}=\frac{\rho_{a i r} v_{\perp} 2 r_{B}}{\mu_{a i r}} .
$$

The diffusion coefficient $D_{g}\left(D_{\text {air }}\right)$ of the interior gas (air) is expressed as

$$
\begin{aligned}
D_{g} & =\frac{1}{3} \tau_{g, \text { col }} v_{g}{ }^{2}, \\
D_{a i r} & =\frac{1}{3} \tau_{a i r, c o l} v_{a i r}{ }^{2}
\end{aligned}
$$

where $\tau_{g, c o l}\left(\tau_{a i r, c o l}\right)$ and $v_{g}\left(v_{a i r}\right)$ are the mean duration of a collision and the mean velocity of a gas (air) molecule, respectively:

$$
\begin{aligned}
& \tau_{g, c o l}=\frac{1}{16 \pi n_{\text {in }} r_{g}^{2}} \sqrt{\frac{\pi m_{g a s, m o l}}{k_{B} T_{g}}}, \\
& \tau_{a i r, c o l}=\frac{1}{16 \pi n_{\text {in }} r_{a i r}^{2}} \sqrt{\frac{\pi m_{a i r, m o l}}{k_{B} T_{a i r, i n}}}, \\
& v_{g}=\sqrt{\frac{8 k_{B} T_{g}}{\pi m_{\text {gas }, \text { mol }}}}, \\
& v_{a i r}=\sqrt{\frac{8 k_{B} T_{a i r, i n}}{\pi m_{a i r, m o l}}}
\end{aligned}
$$

where $k_{B}$ is Boltzmann constant, $n_{\text {in }}$ is the number density inside the balloon, and $r_{g}\left(r_{a i r}\right)$ is the mean radius of the gas (air) molecule taking part in the collision process. In this calculation $r_{g}\left(r_{a i r}\right)$ is set to $0.1 \mathrm{~nm}(0.2 \mathrm{~nm}) . T_{a i r, i n}$ is temperature of the interior air, and is assumed equal $T_{g}$.

The cross section of the duct $S_{D}$ is considered to attain its maximum value $S_{D_{\max }}$, when the balloon is at maximum volume, and to become smaller $\left(S_{D_{\text {small }}}\right)$ when the balloon is shrinking. In this calculation,

$$
S_{D_{\text {small }}}=\frac{S_{D_{\max }}}{8}
$$

which gives a result consistent with the observations concerning the altitude change after the auto-ballast control ceased to operate.

Followings are the characteristics of the balloon used in this calculation:

$\begin{array}{lll}V_{\text {full }} & \text { (maximum volume) } & : 14,671 \mathrm{~m}^{3}, \\ d_{s} & \text { (thickness of the film) } & : 17.8 \mu \mathrm{m}, \\ S_{B} & \text { (surface area of the film) } & : 2,967 \mathrm{~m}^{2}, \\ l_{D} & \text { (length of the venting duct) } & : 6.0 \mathrm{~m}, \\ S_{D} & \text { (cross section of the venting duct) } & : 0.9 \mathrm{~m}^{2} .\end{array}$




\section{REFERENCES}

Carlson, L. A. and W. J. Horn, A new thermal and trajectory model for high altitude balloons, AIAA Paper, No.81-1926, 1981.

Hartmann, D. L., V. Ramanathan, A. Berroir, and G. E. Hunt, Earth radiation budget data and climate research, Rev. Geophys., 24, 2, 439-468, 1986.

Kadokura, A., M. Ejiri, S. Ohta, and H. Akiyama, Polar Patrol Balloon (PPB) experiment of the 30th Japanese Antarctic Research Expedition (1989-1990), Antarct. Rec., 35, 143-154, 1991.

Kiser, C. C., Balloon material testing for Texas A\&M University, Unnumbered Memo to Texas A\&M University from NASA/Langley Research Center, February 26, 1980.

Kreith, F. and J. F. Kreider, Numerical prediction of the performance of high altitude balloons, NCAR Tech. Note, NCAR-IN/STR-65, February, 1974.

Lally, V. E., The radiation controlled balloon (RACOON), Adv. Space Res., 3(6), 19-24, 1983.

Landau, L. D. and E. M. Lifshitz, Fluid Dynamics, edited in Moscow, 1954.

Nishimura, J., M. Fujii, and T. Yamagami, Temperature effect on the balloon motion in the upper atmosphere, Report of the Institute of Space and Aeronautical Science of Tokyo Univ., 9, 1, (B), pp. 167-185, 1973 (in Japanese).

Nishimura, J., Hunting phenomena of the balloon motions observed over Antarctica, ISAS Report, 27, Special Issue for Scientific Ballooning, 3-12, 1990 (in Japanese).

Yamanaka, M. D., K. Yamazaki, and H. Kanzawa, Studies of middle atmosphere dynamics under the polar patrol balloon (PPB) project: Present status and future plans, Proc. NIPR Symp. Upper Atmos. Phys., 1, 65-74, 1988. 